Preprint typeset in JHEP style - HYPER VERSION

\title{
Classification of Supersymmetric Lattice Gauge Theories by Orbifolding
}

\author{
Poul H. Damgaard and So Matsuura \\ The Niels Bohr Institute, The Niels Bohr International Academy, Blegdamsvej 17, \\ DK-2100 Copenhagen, Denmark
}

\begin{abstract}
We provide a general classification of supersymmetric lattice gauge theories that can be obtained from orbifolding of theories with four and eight supercharges. We impose at least one preserved supercharge on the lattice and Lorentz invariance in the naive continuum limit. Starting with four supercharges, we obtain one two-dimensional lattice gauge theory, identical to the one already given in the literature. Starting with eight supercharges, we obtain a unique three-dimensional lattice gauge theory and infinitely many two-dimensional lattice theories. They can be classified according to seven distinct groups, five of which have two preserved supercharges while the others have only one.
\end{abstract}




\section{Contents}

1. Introduction 1

2. Target theories with four supercharges 3

3. Target theories with eight supercharges 9

3.1 Three-dimensional Lattice $(d=3)$

3.2 Two-dimensional Lattice $(d=2)$

$3.2 .1 \quad N_{\text {shift }}=2$

$3.2 .2 \quad N_{\text {shift }}=3$

R.2.3 Relation between $N_{\text {shift }}=2$ and $N_{\text {shift }}=3$

4. Conclusions 22

A. Useful Formulae 23

B. Supersymmetry Transformations 24

B.1 Supersymmetry transformations of the models 1-2 and 2-1 25

B.2 Supersymmetry transformations of the models 1-3 and 2-2

\section{Introduction}

Recently, there has been substantial progress in the formulation of euclidean lattice gauge theories with remnants of space-time supersymmetry [1]-21]. A common feature of almost all of these new approaches has been the connection to topological field theory through twisting. The remnants of supersymmetry that are preserved on the lattice do not generate Poincaré invariance and are thus not in conflict with the reduced lattice group of spacetime symmetries. Rather, the generators are nilpotent operators, completely analogous to the BRST and anti-BRST operators of the corresponding topological field theories in the continuum. Only these BRST and/or anti-BRST symmetries are preserved on the lattice. However, if the naive continuum limit yields the usual twisted formulations of the supersymmetric theories in question it is hoped that the BRST/anti-BRST symmetries are sufficient to guarantee that this occurs at the full quantum level of the lattice theories as well.

A systematic approach to these new formulations of supersymmetric lattice gauge theories is based on the orbifolding technique [1]-[5]. The idea is, roughly, to start with a huge gauge group, say $U\left(k N^{d}\right)$ in a "mother theory" that is dimensionally reduced to zero dimensions. With no space-time coordinates present all fields are really just matrix variables 
living in the adjoint representation of the gauge group. The lattice itself is now generated out of these matrices by means of an orbifold projection followed by deconstruction [22], that is, shifts of the fields. These shifts introduce a basic lattice spacing $a$ in dimensionful units. The aim is typically a $d$-dimensional lattice theory in a finite volume $N^{d}$ and with gauge group $U(k)$. A scheme for doing this on lattices with a continuous time variable was first presented in ref. [1]. A few years ago, Cohen, Kaplan, Katz and Unsal [2]- [4] showed how to extent this procedure to the euclidean formulation. For a detailed analysis of the orbifold construction of supersymmetric lattice theories, see [23]-[26] (see also [27]). For a very nice review of the orbifold construction of lattice theories we refer to [28].

Because supersymmetry requires a careful balance of bosonic and fermionic degrees of freedom, it is evident that lattice prescriptions of such theories must somehow get around the usual fermion doubling problem. This must hold both at finite lattice spacing $a$ and in the continuum limit. As stressed in refs. [6]-11] and [12] 15], one clue seems to lie in a underlying connection to the Dirac-Kähler formulation [29, 30] ${ }^{1}$. Alternatively, the twisting that turns ordinary gauge field theories in the continuum into topological field theories in the continuum requires a departure from the assignment of spin and statistics which is imposed by the spin-statistics theorem. By an "untwisting" in the continuum the usual multiplet of fields that is in accordance with the spin-statistics theorem is recovered. This untwisting is required also for extracting observables from the corresponding lattice theories.

Related to the delicate balance of fermionic and bosonic degrees of freedom is the obvious difficulty of reconciling the conventionally used compact gauge link variables on the lattice with the fermionic partners. In a sense, supersymmetry balances the "zero" of fermionic integrations with an "infinity" coming from non-compact bosonic integrations. In Sugino's approach [16-19], which has compact gauge variables, the supersymmetry transformations are modified on the lattice, even in the case of the topological, nilpotent, symmetries. On the orbifolded lattices, gauge field variables are simply non-compact from the outset, and thus unusual from the lattice perspective. Such an assignment is however perfectly natural if one sees the (always non-compact) scalar fields as dimensionally reduced components of gauge potentials.

Our aim in this paper is to systematically explore the supersymmetric lattice theories that can be generated by orbifold projections. In doing so we shall also provide the answers to the following questions:

- Given a mother theory with a given number of supercharges, how many different lattice theories can be generated at fixed space-time dimension $d$ and fixed number of scalar supercharges on those given lattices?

- Which of those orbifolded lattices lead to Lorentz invariant theories in the naive continuum limit?

\footnotetext{
${ }^{1}$ For two-dimensional $\mathcal{N}=(2,2)$ supersymmetric gauge theory, Suzuki and Taniguchi have given a lattice formulation without this connection [31], arguing that because of super-renormalizability only a single one-loop counterterm needs to be adjusted in order to regain supersymmetry.
} 
- How does the lattice theory depend on the number of field variables that are shifted after the initial orbifold projection?

Remarkably, the classification of supersymmetric lattice theories based on orbifold projections turns out to be relatively simple. Some of the supersymmetric lattice gauge theories that can be generated by this technique have already been described in the literature, but not all. In this paper we shall provide what we believe is the complete classification of orbifolded lattice theories with four and eight supercharges. The classification of theories with sixteen supercharges is a bit more involved, and will be presented in a separate publication [32].

\section{Target theories with four supercharges}

We will begin by briefly recalling the main ingredients in the construction. The starting point is a mother theory which lives in zero space-time dimensions. For a theory with four supercharges we can obtain it by dimensional reduction of $\mathcal{N}=1$ supersymmetric Yang-Mills theory in four euclidean dimensions. As in [2], we take the gauge group to be $U\left(k N^{2}\right)$, in anticipation of at most two-dimensional lattices in this case. The restriction to $U\left(k N^{2}\right)$ rather than $S U\left(k N^{2}\right)$ (or other gauge groups) is not essential. After dimensional reduction the mother theory takes the form

$$
S_{\mathrm{m}}=\frac{1}{g^{2}} \operatorname{Tr}\left(-\frac{1}{4}\left[v_{\alpha}, v_{\beta}\right]^{2}+\frac{i}{2} \bar{\Psi} \Gamma_{\alpha}\left[v_{\alpha}, \Psi\right]\right), \quad(\alpha, \beta=0, \cdots, 3)
$$

where $\Gamma_{\alpha}$ are $S O(4)$ Dirac matrices, $v_{\alpha}$ are $k N^{2} \times k N^{2}$ hermitian matrices, $\Psi$ is a fourcomponent fermion and $\bar{\Psi} \equiv \Psi^{T} C$ with the charge conjugation matrix $C$ satisfying,

$$
C^{-1} \Gamma_{\alpha} C=-\Gamma_{\alpha}^{T}
$$

Following [2], we choose a chiral representation of the $\gamma$-matrices,

$$
\Gamma_{\alpha}=\left(\begin{array}{cc}
0 & \sigma_{\alpha} \\
\bar{\sigma}_{\alpha} & 0
\end{array}\right)
$$

with $\sigma_{\alpha}=\left(\mathbf{1},-i \tau_{i}\right)$ and $\bar{\sigma}_{\alpha}=\left(\mathbf{1}, i \tau_{i}\right)$. the charge conjugation matrix is then represented as

$$
C=\Gamma_{0} \Gamma_{2}=\left(\begin{array}{cc}
i \tau_{2} & 0 \\
0 & -i \tau_{2}
\end{array}\right)
$$

It is convenient to decompose the four-spinors into the two-component chiral components as follows:

$$
\Psi \equiv\left(\begin{array}{c}
\Psi^{(1)} \\
\Psi^{(2)}
\end{array}\right) \quad, \quad \Psi^{(1)} \equiv\left(\begin{array}{c}
\chi_{12} \\
\eta
\end{array}\right) \quad, \quad \Psi^{(2)} \equiv\left(\begin{array}{c}
\psi_{2} \\
\psi_{1}
\end{array}\right)
$$

Introducing the complex combinations

$$
z_{1} \equiv v_{1}+i v_{2},
$$




$$
z_{2} \equiv v_{0}+i v_{3}
$$

the action (2.1) takes the form

$$
S_{\mathrm{m}}=\frac{1}{g^{2}} \operatorname{Tr}\left(\frac{1}{4}\left|\left[z_{m}, z_{n}\right]\right|^{2}+\frac{1}{8}\left[z_{m}, \bar{z}_{m}\right]^{2}+\psi_{m}\left[\bar{z}_{m}, \eta\right]-\chi_{m n}\left[z_{m}, \psi_{n}\right]\right)
$$

with $m, n=1,2$ and $\chi_{m n}=-\chi_{n m}$.

The next step is to identify the maximal number of $U(1)$-symmetries. Because the mother theory is obtained from a four-dimensional euclidean field theory, it has inherited the associated $S O(4)$ Lorentz symmetry. In addition, the fermionic part of the action is invariant under $U(1)$ chiral rotations. The maximal set of $U(1)$-symmetries is therefore $U(1)^{3}$, and we can choose them as $S O(2)_{12} \times S O(2)_{03} \times U(1)$, where the indices refer to the corresponding planes of the original four-dimensional theory. We denote the abelian charges associated with these three $U(1)$ symmetries by $q_{1}, q_{2}$ and $q_{3}$, respectively. To identify the charges of individual fermionic components one notes that the generators of the original $S O(4)$ rotation symmetries are given by the commutator,

$$
\Gamma_{\alpha \beta}=\frac{i}{4}\left(\Gamma_{\alpha} \Gamma_{\beta}-\Gamma_{\beta} \Gamma_{\alpha}\right)=\left(\begin{array}{cc}
\sigma_{\alpha \beta} & 0 \\
0 & \bar{\sigma}_{\alpha \beta}
\end{array}\right)
$$

where

$$
\sigma_{\alpha \beta}=\frac{i}{4}\left(\sigma_{\alpha} \bar{\sigma}_{\beta}-\sigma_{\beta} \bar{\sigma}_{\alpha}\right), \quad \bar{\sigma}_{\alpha \beta}=\frac{i}{4}\left(\bar{\sigma}_{\alpha} \sigma_{\beta}-\bar{\sigma}_{\beta} \sigma_{\alpha}\right)
$$

The generator of rotations in the 12-plane is thus

$$
\Gamma_{12}=\left(\begin{array}{cc}
-\frac{1}{2} \tau_{3} & 0 \\
0 & -\frac{1}{2} \tau_{3}
\end{array}\right)
$$

while the generator of rotations in the 03-plane is

$$
\Gamma_{03}=\left(\begin{array}{cc}
-\frac{1}{2} \tau_{3} & 0 \\
0 & \frac{1}{2} \tau_{3}
\end{array}\right)
$$

We can now fill in the table of $U(1)$ charges. The original symmetry on the fermions $\Psi$ and $\bar{\Psi}$ corresponds to equal charges $q_{3}=+1 / 2$ for the left chiral components $\chi_{12}$ and $\eta$, and $q_{3}=-1 / 2$ for the right chiral components $\psi_{m}$. The two other $U(1)$ charges follow by acting with the generators shown above. Supplemented with the corresponding $S O(2)$ charges for the complex vector fields $z_{m}$ and $\bar{z}_{m}$ this leads to the charge assignments of Table 1 .

The mother theory has four supercharges. To ensure at least one unbroken supersymmetry in the orbifolded theory we need at least one fermion that transforms as a singlet under the $U(1)$ symmetries. This may be intuitively clear from the fact that we precisely wish to keep those supersymmetry charges that will transform trivially under the reduced set of Poincaré symmetries compatible with the generated lattice. A more direct argument has been given in ref. [2]. We choose the singlet fermion to be $\eta$. As can be seen from 
Table 1: The charge assignment of the maximal $U(1)$ symmetries

\begin{tabular}{c|cccccc} 
& $z_{1}$ & $z_{2}$ & $\eta$ & $\chi_{12}$ & $\psi_{1}$ & $\psi_{2}$ \\
\hline$q_{1}$ & 1 & 0 & $1 / 2$ & $-1 / 2$ & $-1 / 2$ & $1 / 2$ \\
$q_{2}$ & 0 & 1 & $1 / 2$ & $-1 / 2$ & $1 / 2$ & $-1 / 2$ \\
$q_{3}$ & 0 & 0 & $1 / 2$ & $1 / 2$ & $-1 / 2$ & $-1 / 2$
\end{tabular}

Table 1 , the $\eta$ is unique in having all $q_{i}$ 's equal, while the three other fermions have two $q_{i}$ 's of $-1 / 2$ and one $q_{i}$ of $+1 / 2$. One might therefore expect two classes of lattice theories, depending on whether $\eta$ or one of $\chi_{12}, \psi_{m}$ is taken to be a scalar under these $U(1)$ symmetries. However, one can easily show that this is not the case. Even if we choose another fermion $\left(\psi_{1}\right.$, for example) to be a singlet, we obtain exactly the same orbifolded action after a renaming of fields. Thus the resulting supersymmetric theory on the abstract orbifolded lattice is actually unique.

Because of the constraint that the $\eta$ must have zero charge, we are left with two free $U(1)$ symmetries under which all fields should have integer charges. In contrast to previous work [2]- [5], we do not insist that these integers be \pm 1 since our purpose is to construct all possible lattice formulations based on orbifolding. As there are just two $U(1)$ charges free after fixing the $\eta$ to have zero charge, we can generate at most two-dimensional lattices in the present case. Let us define two charge combinations,

$$
\begin{aligned}
& r_{1} \equiv \ell_{1}^{1} q_{1}+\ell_{1}^{2} q_{2}-\left(\ell_{1}^{1}+\ell_{1}^{2}\right) q_{3}, \\
& r_{2} \equiv \ell_{2}^{1} q_{1}+\ell_{2}^{2} q_{2}-\left(\ell_{2}^{1}+\ell_{2}^{2}\right) q_{3},
\end{aligned}
$$

for which $\eta$ automatically has vanishing charge. It is then convenient to introduce two vectors,

$$
\mathbf{e}_{1} \equiv\left(\begin{array}{l}
\ell_{1}^{1} \\
\ell_{2}^{1}
\end{array}\right) \quad, \quad \mathbf{e}_{2} \equiv\left(\begin{array}{l}
\ell_{1}^{2} \\
\ell_{2}^{2}
\end{array}\right)
$$

so that the charge assignments of Table 1 generalize to the simple form given in Table 2 . Here, since we are interested in obtaining at least a two-dimensional theory, we assume that $\mathbf{e}_{1}$ and $\mathbf{e}_{2}$ are linearly independent. In other words, we can uniquely express any two-dimensional vector $\mathbf{k} \in \mathbb{Z}^{2}$ as

$$
\mathbf{k}=\sum_{m=1,2} k_{m} \mathbf{e}_{m} . \quad\left(k_{m} \in \mathbb{Z}\right)
$$

Table 2: The two remaining $U(1)$ charges

\begin{tabular}{c|cccccc} 
& $z_{1}$ & $z_{2}$ & $\eta$ & $\chi_{12}$ & $\psi_{1}$ & $\psi_{2}$ \\
\hline $\mathbf{r}$ & $\mathbf{e}_{1}$ & $\mathbf{e}_{2}$ & $\mathbf{0}$ & $-\mathbf{e}_{1}-\mathbf{e}_{2}$ & $\mathbf{e}_{1}$ & $\mathbf{e}_{2}$
\end{tabular}

Based on these two remaining $U(1)$ symmetries we can now carry out the orbifold projection. As explained in detail in refs. [2, 28] this makes use of a $Z_{N} \times Z_{N}$ subgroup 
of $U(1) \times U(1)$. One projects out all field components that are not rendered invariant by the action of this $Z_{N} \times Z_{N}$ symmetry. In Appendix A we collect some useful formulas for performing this projection. It can be summarized by an expansion of all fields in terms of variables living on an abstract lattice labelled by two-vectors $\mathbf{k}$ :

$$
\begin{aligned}
z_{m} & =\sum_{\mathbf{k}} z_{m}(\mathbf{k}) \otimes E_{\mathbf{k}, \mathbf{k}+\mathbf{e}_{m}} \\
\bar{z}_{m} & =\sum_{\mathbf{k}} \bar{z}_{m}(\mathbf{k}) \otimes E_{\mathbf{k}+\mathbf{e}_{m}, \mathbf{k}} \\
\eta & =\sum_{\mathbf{k}} \eta(\mathbf{k}) \otimes E_{\mathbf{k}, \mathbf{k}} \\
\psi_{m} & =\sum_{\mathbf{k}} \psi_{m}(\mathbf{k}) \otimes E_{\mathbf{k}, \mathbf{k}+\mathbf{e}_{m}} \\
\chi_{12} & =\sum_{\mathbf{k}} \chi_{12}(\mathbf{k}) \otimes E_{\mathbf{k}+\mathbf{e}_{1}+\mathbf{e}_{2}, \mathbf{k}}
\end{aligned}
$$

where $z_{m}(\mathbf{k}), \bar{z}_{m}(\mathbf{k})$ and so on are $k \times k$ matrices and $E_{\mathbf{k}, 1}$ is defined by (A.7) in Appendix A. Making use of the orthogonality relation (A.9) for the E's, we arrive at an orbifolded theory described by the abstract lattice action,

$$
\begin{aligned}
S_{\text {orb }}= & \frac{1}{g^{2}} \operatorname{Tr} \sum_{\mathbf{k}}\left(\frac{1}{4}\left|z_{m}(\mathbf{k}) z_{n}\left(\mathbf{k}+\mathbf{e}_{m}\right)-z_{n}(\mathbf{k}) z_{m}\left(\mathbf{k}+\mathbf{e}_{n}\right)\right|^{2}\right. \\
& +\frac{1}{8}\left(z_{m}(\mathbf{k}) \bar{z}_{m}(\mathbf{k})-\bar{z}_{m}\left(\mathbf{k}-\mathbf{e}_{m}\right) z_{m}\left(\mathbf{k}-\mathbf{e}_{m}\right)\right)^{2} \\
& +\psi_{m}(\mathbf{k})\left(\bar{z}_{m}(\mathbf{k}) \eta(\mathbf{k})-\eta\left(\mathbf{k}+\mathbf{e}_{m}\right) \bar{z}_{m}(\mathbf{k})\right) \\
& \left.-\frac{1}{2} \chi_{m n}(\mathbf{k})\left(z_{m}(\mathbf{k}) \psi_{n}\left(\mathbf{k}+\mathbf{e}_{n}\right)-\psi_{n}(\mathbf{k}) z_{m}\left(\mathbf{k}+\mathbf{e}_{n}\right)-(m \leftrightarrow n)\right)\right),
\end{aligned}
$$

where we implicitly sum over repeated indices $m, n=1,2$. The lattice is periodic and of size $N \times N$. Variables $z_{m}(\mathbf{k}), \bar{z}_{m}(\mathbf{k}), \psi_{m}(\mathbf{k})$ transform as bifundamentals of $U(k)$,

$$
z_{m}(\mathbf{k}) \rightarrow V(\mathbf{k})^{\dagger} z_{m}(\mathbf{k}) V\left(\mathbf{k}+\mathbf{e}_{m}\right) \quad, \quad \bar{z}_{m}(\mathbf{k}) \rightarrow V\left(\mathbf{k}+\mathbf{e}_{m}\right)^{\dagger} \bar{z}_{m}(\mathbf{k}) V(\mathbf{k})
$$

while $\eta(\mathbf{k})$ transforms as an adjoint under $U(k)$,

$$
\eta(\mathbf{k}) \rightarrow V(\mathbf{k})^{\dagger} \eta(\mathbf{k}) V(\mathbf{k}),
$$

and finally $\chi_{12}$ also transforms as a bifundamental,

$$
\chi_{12}(\mathbf{k}) \rightarrow V\left(\mathbf{k}+\mathbf{e}_{1}+\mathbf{e}_{2}\right)^{\dagger} \chi_{12}(\mathbf{k}) V(\mathbf{k})
$$

While these transformation rules are similar to those of lattice fields living on sites, links, and corners (or, alternatively, diagonal links), there is yet no space-time lattice, no lattice spacing $a$, and no kinetic energy terms "hopping" between different sites. Just as the mother theory can be viewed as Eguchi-Kawai large- $N$ reduction [33] in the continuum, orbifolding is reminiscent of the similar Eguchi-Kawai reduction in a finite volume [34]. 
We can now see why we only those supersymmetry charges that have vanishing $U(1)$ charges will be preserved on the lattice. In the mother theory we have an exact Leibniz rule for the way supersymmetry charges act on products of fields. Consider now the way a supersymmetry charge will act on the lattice variables...

As shown in ref. [2], shifts of the variables $z_{m}$ (and $\bar{z}_{m}$ ) generate kinetic energy terms for all fields. This is a consequence of the $U(k)$ symmetry which automatically induces covariant derivatives once kinetic terms are introduced for the $z_{m}$ variables. In general, one can shift $N_{\text {shift }}$ variables $z_{m}$, and if we want the target space theory to be Lorentz invariant in $d$ dimensions it is clear that we need $N_{\text {shift }} \geq d$. In the present case there is not much room left (we are not interested in one-dimensional theories), and we choose $N_{\text {shift }}=d=2$. Then, since $z_{m}$ has classical dimension one,

$$
z_{m}(\mathbf{k}) \rightarrow \frac{1}{a_{m}}+z_{m}(\mathbf{k})
$$

for $m=1,2$. Here the $a_{m}$ 's have the dimension of length. After the shifts (2.20) the action (2.16) takes the following form:

$$
\begin{aligned}
S_{\text {lat }}^{d=2, N=2}= & \frac{1}{g^{2}} \operatorname{Tr} \sum_{\mathbf{k}}\left(\frac{1}{4}\left|\nabla_{m}^{+} z_{n}(\mathbf{k})-\nabla_{n}^{+} z_{m}(\mathbf{k})+z_{m}(\mathbf{k}) z_{n}\left(\mathbf{k}+\mathbf{e}_{m}\right)-z_{n}(\mathbf{k}) z_{m}\left(\mathbf{k}+\mathbf{e}_{n}\right)\right|^{2}\right. \\
& +\frac{1}{8}\left(\nabla_{m}^{+}\left(z_{m}(\mathbf{k})+\bar{z}_{m}(\mathbf{k})\right)+z_{m}\left(\mathbf{k}+\mathbf{e}_{m}\right) \bar{z}_{m}\left(\mathbf{k}+\mathbf{e}_{m}\right)-\bar{z}_{m}(\mathbf{k}) z_{m}(\mathbf{k})\right)^{2} \\
& +\psi_{m}(\mathbf{k})\left(\nabla_{m}^{+} \eta(\mathbf{k})-\bar{z}_{m}(\mathbf{k}) \eta(\mathbf{k})+\eta\left(\mathbf{k}+\mathbf{e}_{m}\right) \bar{z}_{m}(\mathbf{k})\right) \\
& \left.+\frac{1}{2} \chi_{m n}(\mathbf{k})\left(\nabla_{m}^{+} \psi_{n}(\mathbf{k})+z_{m}(\mathbf{k}) \psi_{n}\left(\mathbf{k}+\mathbf{e}_{m}\right)-\psi_{n}(\mathbf{k}) z_{m}\left(\mathbf{k}+\mathbf{e}_{n}\right)-(m \leftrightarrow n)\right)\right),
\end{aligned}
$$

where, for an arbitrary function $\phi$, we have introduced the forward difference,

$$
\nabla_{m}^{+} \phi(\mathbf{k})=\frac{1}{a_{m}}\left(\phi\left(\mathbf{k}+\mathbf{e}_{m}\right)-\phi(\mathbf{k})\right) .
$$

Note that the $a_{m}$ 's can take arbitrary complex values; $a_{m} \equiv\left|a_{m}\right| e^{i b_{m}}$ in general. However, the phase factors $e^{i b_{m}}$ can be absorbed by proper $U(1)$ rotations for the fields. Thus we can assume $a_{m} \in \mathbb{R}_{+}$.

It is remarkable that the kinetic terms in (2.21) are defined between nearest neighbors, even though we have not restricted the $U(1)$ charges 2.12 to be just \pm 1 . This is a direct consequence of the deconstruction that is used to create the kinetic terms. If we fix the values of $a_{m}$ 's, the action (2.21) describes the same lattice theory even if we change $\mathbf{e}_{m}$ 's as long as $\mathbf{e}_{1}$ and $\mathbf{e}_{2}$ are linearly independent. The lattice theory obtained from the orbifolding procedure is thus uniquely labelled by 1) the values of $a_{m}$ 's and 2) any linear relation among $\mathbf{e}_{m}$ 's. In fact, the arguments of the fields in this theory can be labelled by a set of integers $\left\{k_{m}\right\}$ as (2.14), which is invariant under a change of basis $\left\{\mathbf{e}_{m}\right\}$.

Next, we must consider the naive continuum limit. To this end, it is convenient to introduce an invertible linear mapping,

$$
f: \quad \mathbf{e}_{m} \mapsto \gamma_{m} a_{m} \hat{\mu}_{m}
$$


where $\gamma_{m}$ are arbitrary positive numbers and $\hat{\mu}_{m}$ 's are unit vectors. This map gives a one-to-one association of a space-time lattice with the abstract lattice space generated by the set $\left\{\mathbf{e}_{m}\right\}$. In particular, the lattice spacings are given by this mapping. An abstract vector $\mathbf{k}$ is thus mapped naturally to a space-time position through

$$
f: \mathbf{k} \mapsto \sum_{m=1,2} k_{m} \gamma_{m} a_{m} \hat{\mu}_{m}
$$

As we shall see, we get a non-trivial restriction on this map by insisting on Lorentz invariance in the naive continuum limit. In order to make our notation simple, we will often, unless there is an obvious ambiguity, use the same notation $\mathbf{k}$ to express the space-time position in the following. The continuum limit is thus defined by ${ }^{2}$

$$
a_{m} \rightarrow 0
$$

so that the difference $(2.22)$ becomes the derivative in the continuum limit, viz.,

$$
\frac{1}{a_{m}}\left(\phi\left(\mathbf{k}+\gamma_{m} a_{m} \hat{\mu}_{m}\right)-\phi(\mathbf{k})\right) \rightarrow \gamma_{m} \hat{\mu}_{m} \cdot \vec{\partial} \phi(\mathbf{k}) .
$$

Finally, we must find a proper set of space-time basis vectors $\left\{\hat{\mu}_{m}\right\}$ and values of $\gamma_{m}$ 's for which the continuum theory is Lorentz invariant. It is sufficient to look at the kinetic term of the bosonic fields,

$$
\frac{1}{4}\left|\nabla_{m}^{+} z_{n}(\mathbf{k})-\nabla_{n}^{+} z_{m}(\mathbf{k})\right|^{2}+\frac{1}{8}\left(\nabla_{m}^{+}\left(z_{m}(\mathbf{k})+\bar{z}_{m}(\mathbf{k})\right)\right)^{2}
$$

By expressing $z_{m}(\mathbf{k})$ and $\bar{z}_{m}(\mathbf{k})$ as

$$
\begin{aligned}
z_{m}(\mathbf{k}) & \equiv S_{m}(\mathbf{k})+i T_{m}(\mathbf{k}), \\
\bar{z}_{m}(\mathbf{k}) & \equiv S_{m}(\mathbf{k})-i T_{m}(\mathbf{k}),
\end{aligned}
$$

the continuum limit of (2.27) can be written as

$$
-S_{m}(\mathbf{x})\left(\gamma_{l} \hat{\mu}_{l} \cdot \vec{\partial}\right)^{2} S_{m}(\mathbf{x})-T_{m}(\mathbf{x})\left[\left(\gamma_{l} \hat{\mu}_{l} \cdot \vec{\partial}\right)^{2} \delta_{m n}-\left(\gamma_{m} \hat{\mu}_{m} \cdot \vec{\partial}\right)\left(\gamma_{n} \hat{\mu}_{n} \cdot \vec{\partial}\right)\right] T_{n}(\mathbf{x})
$$

We immediately identify the $S_{m}$ 's are scalar fields in the continuum limit, and therefore impose

$$
\sum_{m=1}^{2}\left(\gamma_{m} \hat{\mu}_{m} \cdot \vec{\partial}\right)^{2}=l^{2} \partial^{2},
$$

for some constant $l$. This equation is easily solved by $\gamma_{1}=\gamma_{2}=l$ and

$$
\hat{\mu}_{1}=\left(\begin{array}{l}
1 \\
0
\end{array}\right), \quad \hat{\mu}_{2}=\left(\begin{array}{l}
0 \\
1
\end{array}\right),
$$

up to rotations and reflections. The kinetic terms (2.27) then become

$$
-l^{2} S_{m}(\mathbf{x}) \partial^{2} S_{m}(\mathbf{x})-l^{2} T_{m}(\mathbf{x})\left[\partial^{2} \delta_{m n}-\partial_{m} \partial_{n}\right] T_{n}(\mathbf{x}) .
$$




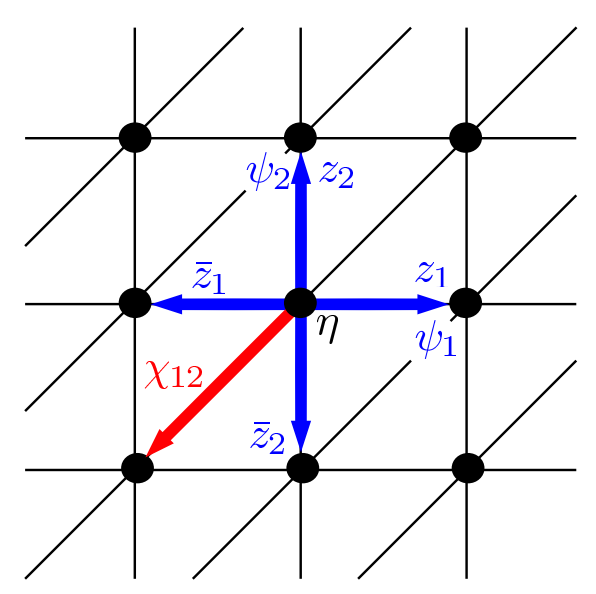

Figure 1: The lattice structure and the field configuration of the theory constructed from the mother theory with four supersymmetries. The lattice is essentially a square lattice and there are also diagonal links. $z_{m}, \bar{z}_{m}, \psi_{m}$ are on the usual link, $\eta$ is on the site and $\chi_{12}$ is on the diagonal link.

The factor $l^{2}$ can be absorbed into the coupling constant by rescaling the fields appropriately, then they are the standard kinetic terms for scalar fields and a gauge field.

One can easily check that the obtained lattice action with the choice of (2.31) is identical to the one given in [2]. Although different $a_{m}$ are allowed, they do not generate theories that are different from the one of the natural choice $a_{1}=a_{2}=a$. Therefore, we conclude that the construction of that paper is the unique orbifolded lattice gauge theory starting from the mother theory (2.1). Different values of $a_{m}$ all give rise to the same naive continuum limit, on account of eq. (2.26), and only redefine what is meant by distance in the different directions. In this formulation, $z_{m}, \bar{z}_{m}, \psi_{m}$ live on links, $\eta$ on sites and $\chi_{12}$ on diagonal links (see Fig. 11). Alternatively, one can think of $\chi_{12}$ as living on corners. As we have seen, in the naive continuum limit the real and imaginary components of $z_{m}$ become two scalar fields and a vector field, respectively. Similarly, the fermion fields build up a two-dimensional Dirac spinor in the continuum limit. As a result, it is expected that four supersymmetries are recovered and the theory becomes two-dimensional $\mathcal{N}=(2,2)$ supersymmetric gauge theory in the continuum limit.

\section{Target theories with eight supercharges}

A theory with eight supercharges is far reacher, and the classification of associated supersymmetric lattice action correspondingly more involved. The mother theory can be obtained by dimensional reduction of six-dimensional $\mathcal{N}=1$ supersymmetric Yang-Mills theory, and in this case we take the gauge group to be $U\left(k N^{d}\right)$ where $d$ can be either 2 or 3 , the maximal dimensionality of the lattice theory. In the notation of [3], it can be written

\footnotetext{
${ }^{2}$ More precisely, we first introduce a common parameter $a$ to set a scale and write $a_{m} \equiv a \beta_{m}$, with $\beta_{m}$ fixed. The continuum limit is then defined by $a \rightarrow 0$.
} 
as

$$
S_{\mathrm{m}}=\frac{1}{g^{2}} \operatorname{Tr}\left(-\frac{1}{4}\left[v_{\alpha}, v_{\beta}\right]^{2}+\bar{\psi} \bar{\Sigma}_{\alpha}\left[v_{\alpha}, \psi\right]\right), \quad(\alpha, \beta=0, \cdots, 5)
$$

where $\bar{\Sigma}_{\alpha}$ are defined through $S O(6)$ Dirac matrices $\Gamma_{\alpha}$ as

$$
\Gamma_{\alpha}=\left(\begin{array}{cc}
0 & \Sigma_{\alpha} \\
\bar{\Sigma}_{\alpha} & 0
\end{array}\right) .
$$

$v_{\alpha}$ are $k N^{d} \times k N^{d}$ hermitian matrices and $\psi$ and $\bar{\psi}$ are independent complex four-component spinors. In the following, we use the representation,

$$
\begin{aligned}
& \bar{\Sigma}_{0}=1_{2} \otimes 1_{2}, \quad \bar{\Sigma}_{1}=-i \tau_{3} \otimes 1_{2}, \quad \bar{\Sigma}_{2}=i \tau_{1} \otimes \tau_{1}, \\
& \bar{\Sigma}_{3}=-i \tau_{1} \otimes \tau_{2}, \quad \bar{\Sigma}_{4}=-i \tau_{1} \otimes \tau_{3}, \quad \bar{\Sigma}_{5}=i \tau_{2} \otimes 1_{2},
\end{aligned}
$$

together with

$$
\begin{aligned}
z_{1} & \equiv v_{0}+i v_{1}, \quad z_{2} \equiv-i\left(v_{2}+i v_{3}\right), \quad z_{3} \equiv-i\left(v_{4}+i v_{5}\right) \\
\bar{z}_{m} & \equiv z_{m}^{\dagger}, \quad(m=1,2,3)
\end{aligned}
$$

and

$$
\psi \equiv\left(\begin{array}{c}
\eta \\
\xi_{23} \\
\xi_{31} \\
\xi_{12}
\end{array}\right), \quad \bar{\psi}^{T} \equiv\left(\begin{array}{c}
-\psi_{1} \\
\chi_{123} \\
\psi_{3} \\
-\psi_{2}
\end{array}\right) .
$$

Then the mother theory can be written as

$$
\begin{aligned}
S_{\mathrm{m}}=\frac{1}{g^{2}}( & \frac{1}{4}\left|\left[z_{m}, z_{n}\right]\right|^{2}+\frac{1}{8}\left[z_{m}, z_{m}\right]^{2} \\
& \left.\quad-\psi_{m}\left[\bar{z}_{m}, \eta\right]+\xi_{m n}\left[z_{m}, \psi_{n}\right]+\frac{1}{2} \chi_{l m n}\left[\bar{z}_{l}, \xi_{m n}\right]\right), \quad(l, m, n=1,2,3)
\end{aligned}
$$

where $\xi_{m n}$ and $\chi_{l m n}$ are completely antisymmetric with respect to the indices.

The next step consists in identifying the maximal set of $U(1)$ symmetries. As explained in ref. [3], the global symmetry of the mother theory is $S O(6) \times S U(2)$ where $S O(6)$ is associated with the Lorentz symmetry of the original six-dimensional theory, and $S U(2)$ is a symmetry which acts only on the fermion fields. We then choose the maximal $U(1)$ symmetry as

$$
S O(6) \times S U(2) \supset S O(2)_{01} \times S O(2)_{23} \times S O(2)_{45} \times U(1),
$$

where $S O(2)_{\alpha \beta}$ is the rotation group in the $(\alpha, \beta)$-plane and $U(1)$ is the Cartan subgroup of $S U(2)$. The configuration of the $U(1)$ charges are summarized in Table. 3 where $q_{1} \cdots q_{4}$ are the $U(1)$ charges corresponding to $S O(2)_{01}, S O(2)_{23}, S O(2)_{45}$ and $U(1)$, respectively.

In order to make an orbifold projection, we need a set of $Z_{N}$ symmetries which are constructed by combining subgroups of the above four $U(1)$ symmetries. As in the previous section, our purpose is to construct all lattice theories which possess at least one scalar 
Table 3: The charge assignment of the maximal $U(1)$ symmetries for the component fields of the mother theory with eight supercharges

\begin{tabular}{c|ccccccccccc} 
& $z_{1}$ & $z_{2}$ & $z_{3}$ & $\eta$ & $\xi_{23}$ & $\xi_{31}$ & $\xi_{12}$ & $\chi_{123}$ & $\psi_{1}$ & $\psi_{2}$ & $\psi_{3}$ \\
\hline$q_{1}$ & 1 & 0 & 0 & $1 / 2$ & $1 / 2$ & $-1 / 2$ & $-1 / 2$ & $1 / 2$ & $1 / 2$ & $-1 / 2$ & $-1 / 2$ \\
$q_{2}$ & 0 & 1 & 0 & $1 / 2$ & $-1 / 2$ & $1 / 2$ & $-1 / 2$ & $1 / 2$ & $-1 / 2$ & $1 / 2$ & $-1 / 2$ \\
$q_{3}$ & 0 & 0 & 1 & $1 / 2$ & $-1 / 2$ & $-1 / 2$ & $1 / 2$ & $1 / 2$ & $-1 / 2$ & $-1 / 2$ & $1 / 2$ \\
$q_{4}$ & 0 & 0 & 0 & $1 / 2$ & $1 / 2$ & $1 / 2$ & $1 / 2$ & $-1 / 2$ & $-1 / 2$ & $-1 / 2$ & $-1 / 2$
\end{tabular}

supersymmetry. We then need at least one fermion that is singlet under the $Z_{N}$ symmetries. We choose it to be $\eta$. Again the result is, after relabelling, the same if we choose another component of fermions as singlet. By this constraint, we can define at most three $U(1)$ symmetries for which all fields have integer charges as

$$
r_{m} \equiv l_{m}^{1} q_{1}+l_{m}^{2} q_{2}+l_{m}^{3} q_{3}-\left(l_{m}^{1}+l_{m}^{2}+l_{m}^{3}\right) q_{4}, \quad\left(m=1,2,3, \quad l_{m}^{n} \in \mathbb{Z}\right)
$$

and we define

$$
\mathbf{e}_{m} \equiv\left(\begin{array}{l}
l_{1}^{m} \\
l_{2}^{m} \\
l_{3}^{m}
\end{array}\right) . \quad(m=1,2,3)
$$

The integer charges for the component fields can be written in the form of a three-vector as

$$
\mathbf{r}=q_{1} \mathbf{e}_{1}+q_{2} \mathbf{e}_{2}+q_{3} \mathbf{e}_{3}-q_{4}\left(\mathbf{e}_{1}+\mathbf{e}_{2}+\mathbf{e}_{3}\right),
$$

which is summarized in Table 4 . Note that we do not assume that the $\mathbf{e}_{m}$ 's are linearly independent. As expected, the dimensionality of the lattice theory is determined by the

\begin{tabular}{|c|c|c|c|c|c|c|c|c|c|c|c|}
\hline & $z_{1}$ & $z_{2}$ & $z_{3}$ & $\eta$ & $\xi_{23}$ & $\xi_{31}$ & $\xi_{12}$ & $\chi_{123}$ & $\psi_{1}$ & $\psi_{2}$ & $\psi_{3}$ \\
\hline $\mathbf{r}$ & $\mathbf{e}_{1}$ & $\mathbf{e}_{2}$ & $\mathbf{e}_{3}$ & 0 & $-\mathbf{e}_{2}-\mathbf{e}_{3}$ & $-\mathbf{e}_{3}-\mathbf{e}_{1}$ & $-\mathbf{e}_{1}-\mathbf{e}_{2}$ & $\mathbf{e}_{1}+\mathbf{e}_{2}+\mathbf{e}_{3}$ & $\mathbf{e}_{1}$ & $\mathbf{e}_{2}$ & $\mathbf{e}_{3}$ \\
\hline
\end{tabular}
number of linearly independent components in $\left\{\mathbf{e}_{m}\right\}$.

Table 4: The remaining three $U(1)$ charges

We can then carry out the orbifold projection by a $Z_{N}^{d}$ subgroup of the remaining three $U(1)$ symmetries. As in the previous section, it can be achieved by expanding all fields by fields living on an abstract lattice labelled by vectors $\mathbf{k}$ :

$$
\begin{aligned}
z_{m} & =\sum_{\mathbf{k}} z_{m}(\mathbf{k}) \otimes E_{\mathbf{k}, \mathbf{k}+\mathbf{e}_{m}} \\
\bar{z}_{m} & =\sum_{\mathbf{k}} \bar{z}_{m}(\mathbf{k}) \otimes E_{\mathbf{k}+\mathbf{e}_{m}, \mathbf{k}} \\
\eta & =\sum_{\mathbf{k}} \eta(\mathbf{k}) \otimes E_{\mathbf{k}, \mathbf{k}}
\end{aligned}
$$




$$
\begin{aligned}
\psi_{m} & =\sum_{\mathbf{k}} \psi_{m}(\mathbf{k}) \otimes E_{\mathbf{k}, \mathbf{k}+\mathbf{e}_{m}} \\
\xi_{m n} & =\sum_{\mathbf{k}} \xi_{m n}(\mathbf{k}) \otimes E_{\mathbf{k}+\mathbf{e}_{m}+\mathbf{e}_{n}, \mathbf{k}} \\
\chi_{123} & =\sum_{\mathbf{k}} \chi_{123}(\mathbf{k}) \otimes E_{\mathbf{k}, \mathbf{k}+\mathbf{e}_{1}+\mathbf{e}_{2}+\mathbf{e}_{3}},
\end{aligned}
$$

where $z_{m}(\mathbf{k}), \bar{z}_{m}(\mathbf{k}), \cdots$ are the fields on the abstract lattice and $E_{\mathbf{k}, \mathbf{l}}$ is defined in Appendix A. Substituting this expansion into the action (3.6), we obtain the action for the orbifolded theory;

$$
\begin{aligned}
S_{\text {orb }}= & \frac{1}{g^{2}} \operatorname{Tr} \sum_{\mathbf{k}}\left(\frac{1}{4}\left|z_{m}(\mathbf{k}) z_{n}\left(\mathbf{k}+\mathbf{e}_{m}\right)-z_{n}(\mathbf{k}) z_{m}\left(\mathbf{k}+\mathbf{e}_{n}\right)\right|^{2}\right. \\
& +\frac{1}{8}\left(z_{m}(\mathbf{k}) \bar{z}_{m}(\mathbf{k})-\bar{z}_{m}\left(\mathbf{k}-\mathbf{e}_{m}\right) z_{m}\left(\mathbf{k}-\mathbf{e}_{m}\right)\right)^{2} \\
& -\psi_{m}(\mathbf{k})\left(\bar{z}_{m}(\mathbf{k}) \eta(\mathbf{k})-\eta\left(\mathbf{k}+\mathbf{e}_{m}\right) \bar{z}_{m}(\mathbf{k})\right) \\
& +\frac{1}{2} \xi_{m n}(\mathbf{k})\left(z_{m}(\mathbf{k}) \psi_{n}\left(\mathbf{k}+\mathbf{e}_{m}\right)-\psi_{n}(\mathbf{k}) z_{m}\left(\mathbf{k}+\mathbf{e}_{n}\right)-(m \leftrightarrow n)\right) \\
& \left.-\frac{1}{2} \chi_{l m n}(\mathbf{k})\left(\bar{z}_{l}\left(\mathbf{k}+\mathbf{e}_{m}+\mathbf{e}_{n}\right) \xi_{m n}(\mathbf{k})-\xi_{m n}\left(\mathbf{k}+\mathbf{e}_{l}\right) \bar{z}_{l}(\mathbf{k})\right)\right) .
\end{aligned}
$$

This orbifolded theory has $U(k)$ "gauge symmetry";

$$
\begin{array}{rlrl}
z_{m}(\mathbf{k}) & \rightarrow V(\mathbf{k})^{\dagger} z_{m}(\mathbf{k}) V\left(\mathbf{k}+\mathbf{e}_{m}\right), & \bar{z}_{m}(\mathbf{k}) & \rightarrow V\left(\mathbf{k}+\mathbf{e}_{m}\right)^{\dagger} \bar{z}_{m}(\mathbf{k}) V(\mathbf{k}), \\
\psi_{m}(\mathbf{k}) & \rightarrow V(\mathbf{k})^{\dagger} \psi_{m}(\mathbf{k}) V\left(\mathbf{k}+\mathbf{e}_{m}\right), & \bar{\psi}_{m}(\mathbf{k}) & \rightarrow V\left(\mathbf{k}+\mathbf{e}_{m}\right)^{\dagger} \bar{\psi}_{m}(\mathbf{k}) V(\mathbf{k}), \\
\eta(\mathbf{k}) & \rightarrow V(\mathbf{k})^{\dagger} \eta(\mathbf{k}) V(\mathbf{k}), & \xi_{m n}(\mathbf{k}) & \rightarrow V\left(\mathbf{k}+\mathbf{e}_{m}+\mathbf{e}_{n}\right)^{\dagger} \xi_{m n}(\mathbf{k}) V(\mathbf{k}), \\
\chi_{123}(\mathbf{k}) & \rightarrow V(\mathbf{k})^{\dagger} \chi_{123}(\mathbf{k}) V\left(\mathbf{k}+\mathbf{e}_{1}+\mathbf{e}_{2}+\mathbf{e}_{3}\right),
\end{array}
$$

with $V(\mathbf{k}) \in U(k)$. Based on the orbifolded action (3.12), we will construct three-dimensional and two-dimensional lattice theories in turn. As mentioned above, the dimensionality of the lattice equals the number of linearly independent vectors in $\left\{\mathbf{e}_{m}\right\}$.

\subsection{Three-dimensional Lattice $(d=3)$}

In this subsection we assume that all $\mathbf{e}_{m}$ are linearly independent so that $\left\{\mathbf{e}_{m}\right\}$ forms a basis of a three-dimensional lattice. As in the previous section, we shift $z_{m}$ (and $\bar{z}_{m}$ ) in order to generate kinetic terms. Since we want to construct a three-dimensional theory, we must shift all three $z_{m}$ so that $\left(N_{\text {shift }}=3\right)$ :

$$
z_{m}(\mathbf{k}) \rightarrow \frac{1}{a_{m}}+z_{m}(\mathbf{k}) . \quad(m=1,2,3)
$$

We can again assume $a_{m} \in \mathbb{R}_{+}$without loss of generality. Then the lattice action becomes

$$
S_{\text {lat }}^{d=3, N=3}=\frac{1}{g^{2}} \operatorname{Tr} \sum_{\mathbf{k}}\left(\frac{1}{4}\left|\nabla_{m}^{+} z_{n}(\mathbf{k})-\nabla_{n}^{+} z_{m}(\mathbf{k})+z_{m}(\mathbf{k}) z_{n}\left(\mathbf{k}+\mathbf{e}_{m}\right)-z_{n}(\mathbf{k}) z_{m}\left(\mathbf{k}+\mathbf{e}_{n}\right)\right|^{2}\right.
$$




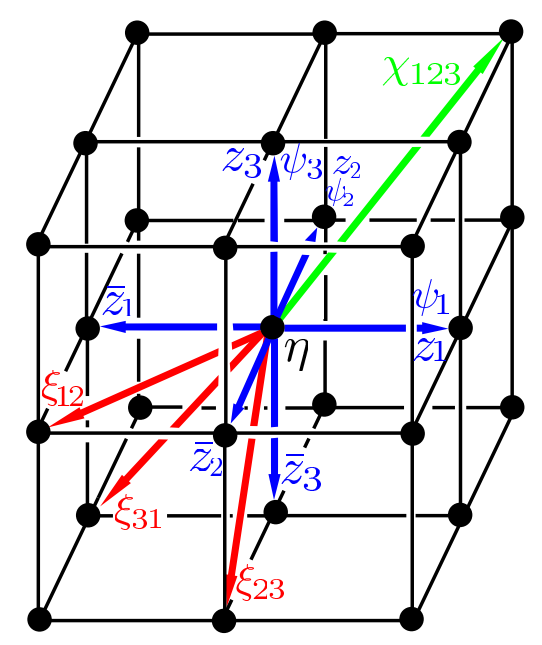

Figure 2: The lattice structure and the field configuration of the three-dimensional lattice formulation.

$$
\begin{aligned}
& +\frac{1}{8}\left(\nabla_{m}^{+}\left(z_{m}(\mathbf{k})+\bar{z}_{m}(\mathbf{k})\right)+z_{m}\left(\mathbf{k}+\mathbf{e}_{m}\right) \bar{z}_{m}\left(\mathbf{k}+\mathbf{e}_{m}\right)-\bar{z}_{m}(\mathbf{k}) z_{m}(\mathbf{k})\right)^{2} \\
& -\psi_{m}(\mathbf{k})\left(\nabla_{m}^{+} \eta(\mathbf{k})-\bar{z}_{m}(\mathbf{k}) \eta(\mathbf{k})+\eta\left(\mathbf{k}+\mathbf{e}_{m}\right) \bar{z}_{m}(\mathbf{k})\right) \\
& +\frac{1}{2} \xi_{m n}(\mathbf{k})\left(\nabla_{m}^{+} \psi_{n}(\mathbf{k})+z_{m}(\mathbf{k}) \psi_{n}\left(\mathbf{k}+\mathbf{e}_{m}\right)-\psi_{n}(\mathbf{k}) z_{m}\left(\mathbf{k}+\mathbf{e}_{n}\right)-(m \leftrightarrow n)\right) \\
& \left.-\frac{1}{2} \chi_{l m n}(\mathbf{k})\left(\nabla_{l}^{+} \xi_{m n}(\mathbf{k})-\bar{z}_{l}\left(\mathbf{k}+\mathbf{e}_{m}+\mathbf{e}_{n}\right) \xi_{m n}(\mathbf{k})+\xi_{m n}\left(\mathbf{k}+\mathbf{e}_{l}\right) \bar{z}_{l}(\mathbf{k})\right)\right) .
\end{aligned}
$$

where the difference operator $\nabla_{m}^{+}$is defined by (2.22). As for the case of the four supercharges, the lattice theory is labelled by the values of $a_{m}$ and the linear relation among $\mathbf{e}_{m}$. Therefore, since the $\mathbf{e}_{m}$ by construction are linearly independent here, the threedimensional lattice theory depends only on the parameters $a_{m}$.

Next we consider the continuum limit. Again, we introduce lattice spacings through the linear map,

$$
f: \mathbf{e}_{m} \mapsto \gamma_{m} a_{m} \hat{\mu}_{m}, \quad(m=1,2,3)
$$

and define the continuum limit by $a_{m} \rightarrow 0$. The difference operator $\nabla_{m}^{+}$becomes a derivative operator $\gamma_{m} \hat{\mu}_{m} \cdot \vec{\partial}$ in that limit. As in the previous section the condition of Lorentz invariance in the continuum limit determines the set $\left\{\hat{\mu}_{m}\right\}$ uniquely as

$$
\hat{\mu}_{1}=\left(\begin{array}{l}
1 \\
0 \\
0
\end{array}\right), \quad \hat{\mu}_{2}=\left(\begin{array}{l}
0 \\
1 \\
0
\end{array}\right), \quad \hat{\mu}_{3}=\left(\begin{array}{l}
0 \\
0 \\
1
\end{array}\right),
$$

up to rotations and reflections, and $\gamma_{1}=\gamma_{2}=\gamma_{3}$. In this way we obtain the action that was constructed in [3] after setting all $a_{m}$ equal. The lattice is cubic and the theory possesses one scalar supersymmetry from the orbifold construction. Variables $z_{m}, \bar{z}_{m}, \psi_{m}$ live on the usual links, $\eta$ on sites, $\xi_{m n}$ on square diagonal links, and $\chi_{123}$ lives on body diagonal 
links (see Fig.2). In the continuum limit, the real and imaginary components of $z_{m}$ become three scalar fields and a vector field, respectively. The eight fermion fields form two threedimensional Dirac spinors. As a result, eight supersymmetries are recovered in the naive continuum limit which turns out to yield three-dimensional supersymmetric gauge theory with 8 supersymmetries [3]. We conclude that (3.15) is the unique three-dimensional lattice action constructed by orbifolding from the mother theory given in (3.1).

\subsection{Two-dimensional Lattice $(d=2)$}

In this subsection, we classify the two-dimensional lattices that can be constructed from the mother theory (3.1). Two dimensional theories appear when there are only two linearly independent vectors in $\left\{\mathbf{e}_{m}\right\}$, which we choose to be $\mathbf{e}_{1}$ and $\mathbf{e}_{2}$. The vector $\mathbf{e}_{3}$ can thus be expressed as a linear combination of these:

$$
\mathbf{e}_{3}=p \mathbf{e}_{1}+q \mathbf{e}_{2} . \quad(p, q \in \mathbb{Q})
$$

The fact that $p$ and $q$ must be rational numbers follows from the quantization of the $U(1)$ charges and the definition of the $\mathbf{e}_{m}$ 's. For the purpose of the future discussion, we assume that $p$ and $q$ satisfy

$$
p q \geq 0 .
$$

Otherwise, we can always swap the roles of $\mathbf{e}_{m}$ so that they satisfy (3.19). The new point in the present case is that we can construct a lattice theory by shifting either two $\left(N_{\text {shift }}=2\right)$ or three $\left(N_{\text {shift }}=3\right)$ of $z_{m}$, and this gives rise to different lattice theories. As we shall see below, one can in fact construct seven distinct groups of lattice theories that all correspond to two-dimensional $\mathcal{N}=(4,4)$ gauge theory in the naive continuum limit. Five of these lattice theories have two conserved supercharges while the remaining two have only one.

3.2.1 $N_{\text {shift }}=2$

We first we consider the case where one shifts only $z_{1}$ and $z_{2}$;

$$
\begin{aligned}
z_{m}(\mathbf{k}) & \rightarrow \frac{1}{a_{m}}+z_{m}(\mathbf{k}), \quad(m=1,2) \\
z_{3}(\mathbf{k}) & \rightarrow z_{3}(\mathbf{k}),
\end{aligned}
$$

with $a_{m} \in \mathbb{R}_{+}(m=1,2)$. Then we obtain the lattice action,

$$
\begin{aligned}
S_{\text {lat }}^{d=2, N=2}= & \frac{1}{g^{2}} \operatorname{Tr} \sum_{\mathbf{k}}\left(\frac{1}{4}\left|\nabla_{m}^{+} z_{n}(\mathbf{k})-\nabla_{n}^{+} z_{m}(\mathbf{k})+z_{m}(\mathbf{k}) z_{n}\left(\mathbf{k}+\mathbf{e}_{m}\right)-z_{n}(\mathbf{k}) z_{m}\left(\mathbf{k}+\mathbf{e}_{n}\right)\right|^{2}\right. \\
& +\frac{1}{8}\left(\nabla_{m}^{+}\left(z_{m}(\mathbf{k})+\bar{z}_{m}(\mathbf{k})\right)+z_{m}\left(\mathbf{k}+\mathbf{e}_{m}\right) \bar{z}_{m}\left(\mathbf{k}+\mathbf{e}_{m}\right)-\bar{z}_{m}(\mathbf{k}) z_{m}(\mathbf{k})\right)^{2} \\
& +\frac{1}{2}\left|\nabla_{m}^{+} z_{3}(\mathbf{k})+z_{m}(\mathbf{k}) z_{3}\left(\mathbf{k}+\mathbf{e}_{m}\right)-z_{3}(\mathbf{k}) z_{m}\left(\mathbf{k}+\mathbf{e}_{3}\right)\right|^{2} \\
& +\frac{1}{8}\left(z_{3}(\mathbf{k}) \bar{z}_{3}(\mathbf{k})-\bar{z}_{3}\left(\mathbf{k}-\mathbf{e}_{3}\right) z_{3}\left(\mathbf{k}-\mathbf{e}_{3}\right)\right)^{2} \\
& -\psi_{m}(\mathbf{k})\left(\nabla_{m}^{+} \eta(\mathbf{k})-\bar{z}_{m}(\mathbf{k}) \eta(\mathbf{k})+\eta\left(\mathbf{k}+\mathbf{e}_{m}\right) \bar{z}_{m}(\mathbf{k})\right) \\
& -\psi_{3}(\mathbf{k})\left(\bar{z}_{3}(\mathbf{k}) \eta(\mathbf{k})-\eta\left(\mathbf{k}+\mathbf{e}_{3}\right) \bar{z}_{m}(\mathbf{k})\right)
\end{aligned}
$$




$$
\begin{gathered}
+\frac{1}{2} \xi_{m n}(\mathbf{k})\left(\nabla_{m}^{+} \psi_{n}(\mathbf{k})+z_{m}(\mathbf{k}) \psi_{1}\left(\mathbf{k}+\mathbf{e}_{n}\right)-\psi_{n}(\mathbf{k}) z_{2}\left(\mathbf{k}+\mathbf{e}_{m}\right)-(m \leftrightarrow n)\right) \\
+\frac{1}{2} \xi_{m 3}(\mathbf{k})\left(\nabla_{m}^{+} \psi_{3}(\mathbf{k})+z_{m}(\mathbf{k}) \psi_{3}\left(\mathbf{k}+\mathbf{e}_{m}\right)-\psi_{3}(\mathbf{k}) z_{m}\left(\mathbf{k}+\mathbf{e}_{3}\right)\right. \\
\left.-z_{3}(\mathbf{k}) \psi_{m}\left(\mathbf{k}+\mathbf{e}_{3}\right)+\psi_{m}(\mathbf{k}) z_{3}\left(\mathbf{k}+\mathbf{e}_{m}\right)\right) \\
-\chi_{123}(\mathbf{k})\left(\epsilon_{m n}\left(\nabla_{m}^{+} \xi_{n 3}-\bar{z}_{m}\left(\mathbf{k}+\mathbf{e}_{n}+\mathbf{e}_{3}\right) \xi_{n 3}(\mathbf{k})+\xi_{n 3}\left(\mathbf{k}+\mathbf{e}_{m}\right) \bar{z}_{m}(\mathbf{k})\right)\right. \\
\left.\left.-\bar{z}_{3}\left(\mathbf{k}+a \hat{\mu}_{1}+a \hat{\mu}_{2}\right) \xi_{12}(\mathbf{k})+\xi_{12}\left(\mathbf{k}+\mathbf{e}_{3}\right) \bar{z}_{3}(\mathbf{k})\right)\right)
\end{gathered}
$$

with an implicit summation over $m, n=1,2$. Again, the lattice theory is labelled by the values of the $a_{m}$ and the linear relation among the vectors $\mathbf{e}_{m}$, i.e., by the values of $p$ and $q$ in (3.18). We thus obtain infinitely many lattice formulations in this case. As we will see below, they can be classified by the number of preserved supersymmetries on the lattice.

We again introduce lattice spacings through a linear mapping,

$$
f: \mathbf{e}_{m} \mapsto \gamma_{m} a_{m} \hat{\mu}_{m}, \quad\left|\hat{\mu}_{m}\right|=1, \quad \gamma_{m} \in \mathbb{R}_{+} \quad(m=1,2)
$$

and define the continuum limit by $a_{m} \rightarrow 0(m=1,2)$ as before. Since $f$ is linear, $\mathbf{e}_{3}$ is mapped to $p \gamma_{1} a_{1} \hat{\mu}_{1}+q \gamma_{2} a_{2} \hat{\mu}_{2}$. In this case, repeating the proof at the end of Section 2 , we can show that the continuum theory can be Lorentz invariant if and only if

$$
\hat{\mu}_{1}=\left(\begin{array}{l}
1 \\
0
\end{array}\right), \quad \hat{\mu}_{2}=\left(\begin{array}{l}
0 \\
1
\end{array}\right),
$$

up to rotations and reflections, and $\gamma_{1}=\gamma_{2}$. This is a square lattice.

Although there are infinitely many lattice formulations labelled by $(p, q)$, we can classify them by the number of remaining supersymmetries. In fact, this number can be enhanced by tuning $\mathbf{e}_{3}$ properly. There are three cases.

$[1-1] \mathbf{e}_{3}=0$

This is the two-dimensional theory constructed in [3], where it was already shown that it possesses two scalar supersymmetries. In fact, looking at Table 4 , We see that $\psi_{3}$ also becomes a scalar fermion by this choice of $\mathbf{e}_{3}$. In this formulation, $z_{3}, \bar{z}_{3}, \eta$ and $\psi_{3}$ live on sites, $z_{m}, \bar{z}_{m}$ and $\psi_{m}(m=1,2)$ live on the usual links, and $\xi_{12}$ and $\chi_{123}$ are on diagonal links (see (a) in Fig. 3). In the continuum limit, the real components of $z_{m}(m=1,2)$ and $z_{3}$ become four real scalar fields, the imaginary components of $z_{m}(m=1,2)$ becomes a vector field, and the fermion fields combine into two two-dimensional Dirac spinors. As a result, as discussed in [3], the continuum theory is expected to be two-dimensional $\mathcal{N}=(4,4)$ supersymmetric gauge theory.

$[1-2] \mathbf{e}_{3}=-\mathbf{e}_{1}\left(\right.$ or $\left.-\mathbf{e}_{2}\right)$

This gives a new lattice formulation of two-dimensional $\mathcal{N}=(4,4)$ supersymmetric gauge theory. As for the case of $\mathbf{e}_{3}=0$, there is an "accidental" enhancement of supersymmetries and there are again two conserved supercharges on the lattice. In fact, looking 


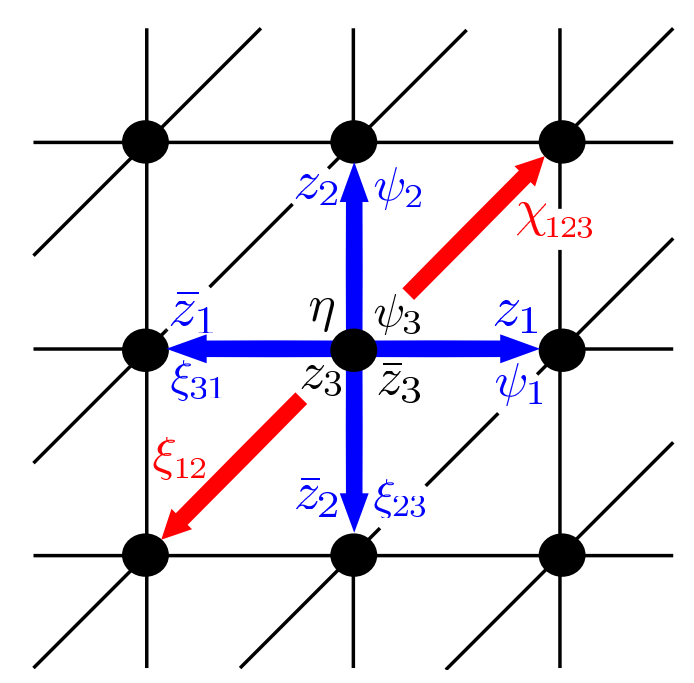

(a) $\mathbf{e}_{3}=0$

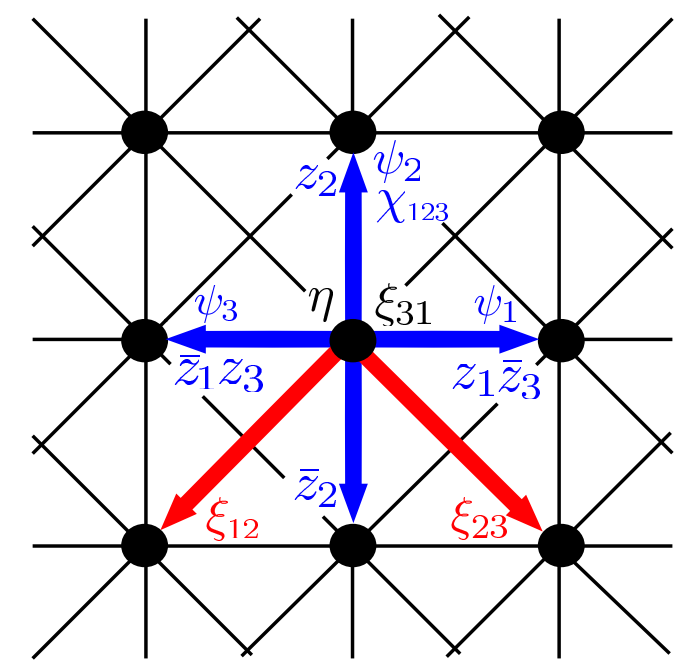

(b) $\mathbf{e}_{3}=-\mathbf{e}_{1}$

Figure 3: The lattice structure and the field configuration of the two-dimensional lattice formulation constructed by shifting $z_{1}$ and $z_{2}$. We have drawn the case of (a) $\mathbf{e}_{3}=0$ and (b) $\mathbf{e}_{3}=-\mathbf{e}_{1}$, where there are two remaining supercharges. Correspondingly, there are two fermions on sites for the both cases.

at Table 4, we see that $\xi_{31}$ (or $\xi_{23}$ ) becomes a singlet under the $U(1)$ transformations. We thus expect on general grounds that there will be two preserved supersymmetries in this case. This can indeed be checked explicitly, both for this case and for the subsequent cases discussed below. We have summarized the proof of this in Appendix B. In this case, $\eta$ and $\xi_{31}$ live on sites, while $z_{m}, \bar{z}_{m}, \psi_{m}(m=1,2,3)$ and $\chi_{123}$ live on the usual links, and $\xi_{12}$ and $\xi_{23}$ sit on diagonal links but in the direction opposite of the case corresponding to $\mathbf{e}_{3}=0$ (see (b) of Fig. 3). The role of the fields in the continuum limit is completely the same as in the case of $\mathbf{e}_{3}=0$, and the continuum theory is again expected to be two-dimensional $\mathcal{N}=(4,4)$ supersymmetric gauge theory.

\section{$[1-3] \mathbf{e}_{1}+\mathbf{e}_{2}+\mathbf{e}_{3}=0$}

This also gives a new lattice formulation of two-dimensional $\mathcal{N}=(4,4)$ supersymmetric gauge theory. In this formulation, $\eta$ and $\chi_{123}$ live on sites, and there thus two remaining supersymmetries as in [1-1] and [1-2] above. The fields $z_{m}, \psi_{m}, \xi_{23}$ and $\xi_{31}(m=1,2)$ live on links, and $z_{3}, \psi_{3}$ and $\xi_{12}$ live on diagonal links (see (c) of Fig. 四). The role of the fields in the naive continuum limit is again the same as in the cases of [1-1] and [1-2], and the continuum theory is expected to be two-dimensional $\mathcal{N}=(4,4)$ supersymmetric gauge theory.

$[1-4] \mathbf{e}_{3} \notin\left\{0,-\mathbf{e}_{1},-\mathbf{e}_{2},-\mathbf{e}_{1}-\mathbf{e}_{2}\right\}$

This is again a new lattice formulation of two-dimensional $\mathcal{N}=(4,4)$ supersymmetric gauge theory, but there is now only one supersymmetry preserved on the lattice at finite 


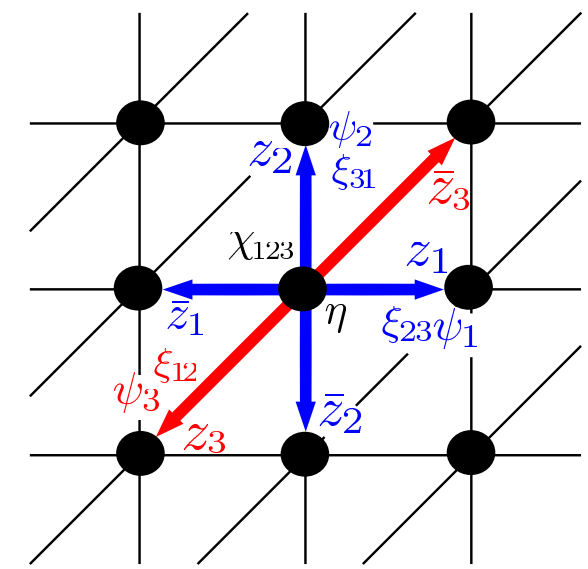

(c) $\mathbf{e}_{1}+\mathbf{e}_{2}+\mathbf{e}_{3}=0$

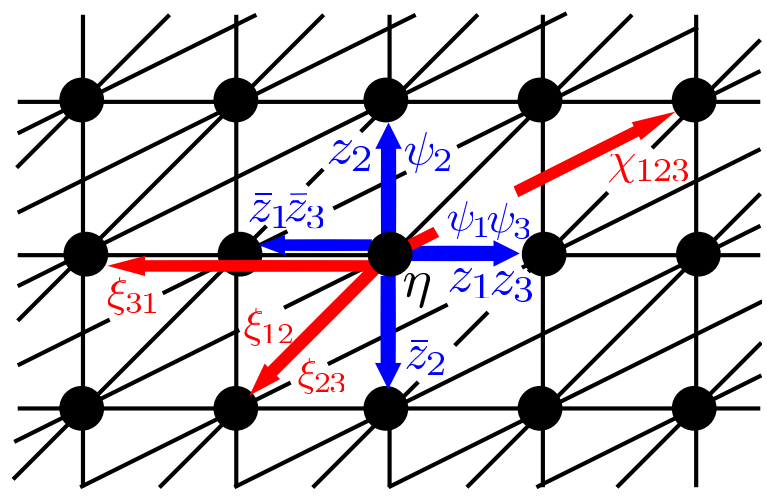

(d) $\mathbf{e}_{3}=\mathbf{e}_{1}$

Figure 4: The lattice structure and the field configuration of two-dimensional lattice formulation constructed by shifting $z_{1}$ and $z_{2}$. We have drawn the cases of (c) $\mathbf{e}_{1}+\mathbf{e}_{2}+\mathbf{e}_{3}=0$ and (d) $\mathbf{e}_{3}=\mathbf{e}_{1}$. There are two remaining supercharges in (c), while there is one supercharge in (d). Comparing to the three cases (a), (b) and (c), both of the lattice structure and the field configuration are less symmetric in the case of $(\mathrm{d})$.

lattice spacing. In this case, the structure of the lattice is less symmetric than the three cases above and there are several kinds of diagonal links in general. We draw the case of $\mathbf{e}_{3}=\mathbf{e}_{1}$ as an example in (d) of Fig. 1 . Although there is less lattice (space-time) symmetry, the roles of the fields in the continuum limit are the same, and the naive continuum theory is again expected to be two-dimensional $\mathcal{N}=(4,4)$ supersymmetric gauge theory.

\subsection{2 $N_{\text {shift }}=3$}

Next, let us consider the case where we shift all $z_{m}$ :

$$
z_{m}(\mathbf{k}) \rightarrow \frac{1}{a_{m}}+z_{m}(\mathbf{k}) . \quad(m=1,2,3)
$$

Then we obtain the action,

$$
\begin{aligned}
S_{\mathrm{lat}}^{d=2, N=3}= & \frac{1}{g^{2}} \operatorname{Tr} \sum_{\mathbf{k}}\left(\frac{1}{4}\left|\nabla_{m}^{+} z_{n}(\mathbf{k})-\nabla_{n}^{+} z_{m}(\mathbf{k})+z_{m}(\mathbf{k}) z_{n}\left(\mathbf{k}+\mathbf{e}_{m}\right)-z_{n}(\mathbf{k}) z_{m}\left(\mathbf{k}+\mathbf{e}_{n}\right)\right|^{2}\right. \\
& +\frac{1}{8}\left(\nabla_{m}^{+}\left(z_{m}(\mathbf{k})+\bar{z}_{m}(\mathbf{k})\right)+z_{m}\left(\mathbf{k}+\mathbf{e}_{m}\right) \bar{z}_{m}\left(\mathbf{k}+\mathbf{e}_{m}\right)-\bar{z}_{m}(\mathbf{k}) z_{m}(\mathbf{k})\right)^{2} \\
& -\psi_{m}(\mathbf{k})\left(\nabla_{m}^{+} \eta(\mathbf{k})-\bar{z}_{m}(\mathbf{k}) \eta(\mathbf{k})+\eta\left(\mathbf{k}+\mathbf{e}_{m}\right) \bar{z}_{m}(\mathbf{k})\right) \\
& +\frac{1}{2} \xi_{m n}(\mathbf{k})\left(\nabla_{m}^{+} \psi_{n}(\mathbf{k})+z_{m}(\mathbf{k}) \psi_{n}\left(\mathbf{k}+\mathbf{e}_{m}\right)-\psi_{n}(\mathbf{k}) z_{m}\left(\mathbf{k}+\mathbf{e}_{n}\right)-(m \leftrightarrow n)\right) \\
& \left.-\frac{1}{2} \chi_{l m n}(\mathbf{k})\left(\nabla_{l}^{+} \xi_{m n}(\mathbf{k})-\bar{z}_{l}\left(\mathbf{k}+\mathbf{e}_{m}+\mathbf{e}_{n}\right) \xi_{m n}(\mathbf{k})+\xi_{m n}\left(\mathbf{k}+\mathbf{e}_{l}\right) \bar{z}_{l}(\mathbf{k})\right)\right)
\end{aligned}
$$


with implicit summation over $l, m, n=1,2,3$. This action is formally of the same form as the three-dimensional lattice theory (3.15), but the interpretation is completely different because now the three vectors $\mathbf{e}_{m}$ span a two-dimensional space-time. As for the above cases, the lattice formulation is labelled by the values of $a_{m}$ 's $(m=1,2,3)$ and the values of $p$ and $q$.

Again, we introduce lattice spacings through the mapping,

$$
f: \mathbf{e}_{m} \mapsto \gamma_{m} a_{m} \hat{\mu}_{m}, \quad\left|\hat{\mu}_{m}\right|=1, \quad(m=1,2,3)
$$

and the continuum limit is defined by $a_{m} \rightarrow 0(m=1,2,3)$. The condition for Lorentz invariance is the same as before,

$$
\sum_{m=1}^{3}\left(\gamma_{m} \hat{\mu}_{m} \cdot \vec{\partial}\right)^{2}=l^{2} \partial^{2}
$$

for some constant $l$. In this case, the condition of Lorentz invariance in the naive continuum limit does not determine $\hat{\mu}_{m}$ uniquely, but determines only the relation between $\hat{\mu}_{m}$ and $\gamma_{m}$. To see this, we write $\hat{\mu}_{m}$ as

$$
\hat{\mu}_{1}=\left(\begin{array}{l}
1 \\
0
\end{array}\right), \quad \hat{\mu}_{2}=\left(\begin{array}{c}
\cos \theta_{2} \\
\sin \theta_{2}
\end{array}\right), \quad \hat{\mu}_{3}=\left(\begin{array}{c}
\cos \theta_{3} \\
\sin \theta_{3}
\end{array}\right) . \quad\left(0 \leq \theta_{2} \leq \theta_{3}<2 \pi\right)
$$

When $\sin \theta_{2}, \sin \theta_{3}$ and $\sin \left(\theta_{3}-\theta_{2}\right)$ are all non-zero, the solutions for $\gamma_{m}$ are

$$
\gamma_{1}^{2}=\frac{l^{2} \cos \theta_{32}}{\sin \theta_{2} \sin \theta_{3}} . \quad \gamma_{2}^{2}=\frac{-l^{2}}{\sin \theta_{32}} \frac{\cos \theta_{3}}{\sin \theta_{2}}, \quad \gamma_{3}^{2}=\frac{l^{2}}{\sin \theta_{32}} \frac{\cos \theta_{2}}{\sin \theta_{3}},
$$

where $\theta_{32} \equiv \theta_{3}-\theta_{2}$. Since the $\gamma_{m}^{2}$ must be positive, the ranges of $\theta_{1}$ and $\theta_{2}$ are restricted to

$$
\begin{gathered}
\frac{\pi}{2}<\theta_{2}<\pi, \quad \theta_{2}+\frac{\pi}{2}<\theta_{3}<\frac{3 \pi}{2} \\
\text { or } \\
\pi<\theta_{2}<\frac{3 \pi}{2}, \quad \frac{3 \pi}{2}<\theta_{3}<\theta_{2}+\frac{\pi}{2}
\end{gathered}
$$

up to rotations and flips of $\hat{\mu}_{m}$. Here, we have required that $\hat{\mu}_{3}$ satisfy the same condition with (3.19), that is, $\hat{\mu}_{3}=\alpha \hat{\mu}_{1}+\beta \hat{\mu}_{2}$ with $\alpha \beta \geq 0$. At the boundary of these regions, we cannot use the formula (3.29) and special care is needed. In this case, we obtain

$$
\begin{array}{rlrl}
\gamma_{1}^{2} & =l^{2}, \gamma_{2}^{2}+\gamma_{3}^{2}=l^{2}, & \left(\theta_{2}, \theta_{3}\right) & =\left(\frac{\pi}{2}, \frac{\pi}{2}\right),\left(\frac{\pi}{2}, \frac{3 \pi}{2}\right),\left(\frac{3 \pi}{2}, \frac{3 \pi}{2}\right) \\
\gamma_{2}^{2}=l^{2}, \gamma_{1}^{2}+\gamma_{3}^{2}=l^{2}, & \left(\theta_{2}, \theta_{3}\right)=\left(\frac{\pi}{2}, \pi\right) \\
\gamma_{3}^{2}=l^{2}, \gamma_{1}^{2}+\gamma_{2}^{2}=l^{2} . & \left(\theta_{2}, \theta_{3}\right)=\left(\pi, \frac{3 \pi}{2}\right)
\end{array}
$$


The situation is different from the previous cases in that there are now restrictions on the values of $a_{m}$. This can be seen as follows. By definition, $f$ maps $\mathbf{e}_{m}$ as

$$
f:\left\{\begin{array}{l}
\mathbf{e}_{1} \mapsto \gamma_{1} a_{1} \hat{\mu}_{1}=\left(\gamma_{1} a_{1}, 0\right) \\
\mathbf{e}_{2} \mapsto \gamma_{2} a_{2} \hat{\mu}_{2}=\left(\gamma_{2} a_{2} \cos \theta_{2}, \gamma_{2} a_{2} \sin \theta_{2}\right) \\
\mathbf{e}_{3} \mapsto \gamma_{3} a_{3} \hat{\mu}_{3}=\left(\gamma_{3} a_{3} \cos \theta_{3}, \gamma_{3} a_{3} \sin \theta_{3}\right)
\end{array}\right.
$$

On the other hand, since $f$ is a linear mapping, the combinations $a_{m} \hat{\mu}_{m}$ must also satisfy

$$
\gamma_{3} a_{3} \hat{\mu}_{3}=p \gamma_{1} a_{1} \hat{\mu}_{1}+q \gamma_{2} a_{2} \hat{\mu}_{2}
$$

Eqs. (3.33) and (3.34) suggest that $a_{2}$ and $a_{3}$ can be solved in terms of $a_{1}$ :

$$
a_{2}=\frac{p}{q} \sqrt{-\frac{\tan \theta_{3}}{\tan \left(\theta_{3}-\theta_{2}\right)}} a_{1}, \quad a_{3}= \pm p \sqrt{\frac{\tan \theta_{2}}{\tan \left(\theta_{3}-\theta_{2}\right)}} a_{1},
$$

where we take plus/minus sign in the case of $(3.30) /(3.31)$, respectively. Since $a_{m} \in \mathbb{R}_{+}$, we see that there is a restriction on the regions of $\left(\theta_{2}, \theta_{3}\right)$ corresponding to the signature of $p$ and $q$; if $p>0$ and $q>0$, we must use (3.30), and if $p<0$ and $q<0$, we must use (3.31) (recall eq. (3.19)). As we shall see, $\theta_{2}$ and $\theta_{3}$ take the values of (3.32) in the cases of $p=0$ or $q=0$.

Conversely, if we impose Lorentz invariance in the naive continuum limit, the linear mapping $f$ is completely determined by given values of $(p, q)$ and $\left\{a_{m}\right\}$ up to the overall factor $l$. In fact, 3.35 can be inverted to give

$$
\tan \theta_{2}=\frac{a_{3}}{q a_{2}} \sqrt{1+\frac{q^{2} a_{2}^{2}+a_{3}^{2}}{p^{2} a_{1}^{2}}}, \quad \tan \theta_{3}=-\frac{q a_{2}}{a_{3}} \sqrt{1+\frac{q^{2} a_{2}^{2}+a_{3}^{2}}{p^{2} a_{1}^{2}}}
$$

which determines $\theta_{2}$ and $\theta_{3}$ uniquely. The values of $\gamma_{m}$ are also determined through the relation $(3.29)$ as

$$
\begin{aligned}
& \gamma_{1}^{2}=\frac{q^{2} a_{2}^{2}+a_{3}^{2}}{p^{2} a_{1}^{2}+q^{2} a_{2}^{2}+a_{3}^{2}} l^{2}, \\
& \gamma_{2}^{2}=\frac{p^{2} q^{2} a_{1}^{2} a_{2}^{2}+a_{3}^{2}\left(p^{2} a_{1}^{2}+q^{2} a_{2}^{2}+a_{3}^{2}\right)}{\left(q^{2} a_{2}^{2}+a_{3}^{2}\right)\left(p^{2} a_{1}^{2}+q^{2} a_{2}^{2}+a_{3}^{2}\right)} l^{2}, \\
& \gamma_{3}^{2}=\frac{p^{2} a_{1}^{2} a_{3}^{2}+q^{2} a_{2}^{2}\left(p^{2} a_{1}^{2}+q^{2} a_{2}^{2}+a_{3}^{2}\right)}{\left(q^{2} a_{2}^{2}+a_{3}^{2}\right)\left(p^{2} a_{1}^{2}+q^{2} a_{2}^{2}+a_{3}^{2}\right)} l^{2} .
\end{aligned}
$$

In particular, the formulae (3.36) and (3.37) can be applied to the cases of $p=0$ or $q=0$, which give (3.32) as announced. We exclude the case of $p=q=0$ here since it leads to a vanishing $\gamma_{3}$. We will discuss this point in the next subsection.

In summary, we can conclude that the lattice formulation for $N_{\text {shift }}=3$ is labelled by the values of $p, q$ and a set of $\left\{a_{m}\right\}$. The spacetime interpretation of the lattice is given through the linear mapping (3.26) in which the values of $\left(\theta_{1}, \theta_{2}\right)$ and $\gamma_{m}$ are determined by (3.36) and (3.37). Although there are infinitely many theories, we can again 
classify them by the number of the remaining supersymmetries. As we shall see, they give different lattice formulations of what in the continuum becomes two-dimensional $\mathcal{N}=(4,4)$ supersymmetric gauge theory.

$[2-1] \mathbf{e}_{3}=-\mathbf{e}_{1}\left(\right.$ or $\left.-\mathbf{e}_{2}\right)$

This is the case of $(p, q)=(-1,0)$ (or $(0,-1))$ and we assume that all $a_{m}$ have finite values. From (3.36), we see $\left(\theta_{2}, \theta_{3}\right)=(\pi / 2,-\pi)$ (or $\left.(\pi / 2,3 \pi / 2)\right)$, then the lattice is a square lattice. The values of $\gamma_{m}$ are given by

$$
\left(\gamma_{1}^{2}, \gamma_{2}^{2}, \gamma_{3}^{2}\right)=\left(\frac{a_{3}^{2} l^{2}}{a_{1}^{2}+a_{3}^{2}}, l^{2}, \frac{a_{1}^{2} l^{2}}{a_{1}^{2}+a_{3}^{2}}\right) . \quad\left(\text { or }\left(l^{2}, \frac{a_{3}^{2} l^{2}}{a_{2}^{2}+a_{3}^{2}}, \frac{a_{2}^{2} l^{2}}{a_{2}^{2}+a_{3}^{2}}\right)\right)
$$

Although the action is different, the field configuration of this theory is the same with [1-2] and thus there are two conserved supercharges. (See (b) of Fig. 3.)

The roles of the fields in the continuum limit are slightly non-trivial. To see this, it is again useful to look at the kinetic terms of $z_{m}$ in the continuum limit,

$$
-l^{2} S_{m}(\mathbf{x}) \partial^{2} S_{m}(\mathbf{x})-T_{m}(\mathbf{x})\left[l^{2} \partial^{2} \delta_{m n}-\left(\gamma_{m} \hat{\mu}_{m} \cdot \vec{\partial}\right)\left(\gamma_{n} \hat{\mu}_{n} \cdot \vec{\partial}\right)\right] T_{n}(\mathbf{x}),
$$

where $l, m, n=1,2,3$, and $S_{m}$ and $T_{m}$ are the real and imaginary components of $z_{m}$, respectively. We then define an orthogonal matrix $P_{m n}$ [4],

$$
\sum_{n=1}^{3} P_{m n} \gamma_{n} \hat{\mu}_{n}= \begin{cases}\left(\begin{array}{l}
l \\
0
\end{array}\right), & (m=1) \\
\left(\begin{array}{l}
0 \\
l
\end{array}\right), & (m=2) \\
0, & (m=3)\end{cases}
$$

with the help of which we can rewrite $T_{m}$ as

$$
T_{m} \equiv \sum_{\mu=1,2} \frac{1}{l}\left(P_{m \mu} A_{\mu}\right)+\frac{1}{l} P_{m 3} S_{4}
$$

Finally (3.39) can be rewritten as

$$
-\sum_{a=1}^{4} S_{a}(\mathbf{x}) \partial^{2} S_{a}(\mathbf{x})-\sum_{\mu, \nu=1}^{2} A_{\mu}(\mathbf{x})\left[\partial^{2} \delta_{\mu \nu}-\partial_{\mu} \partial_{\nu}\right] A_{\nu}(\mathbf{x})
$$

where we have also rescaled $S_{m} \rightarrow \frac{1}{l} S_{m}$. This is nothing but the canonical kinetic terms for scalar bosons and a gauge vector. As a result, the continuum limit of this theory has four real scalar fields and one vector field. One can also show that the kinetic terms of fermions and the interaction terms become that of two-dimensional $\mathcal{N}=(4,4)$ supersymmetric gauge theory in the naive continuum limit.

$[2-2] \mathbf{e}_{1}+\mathbf{e}_{2}+\mathbf{e}_{3}=0$ 


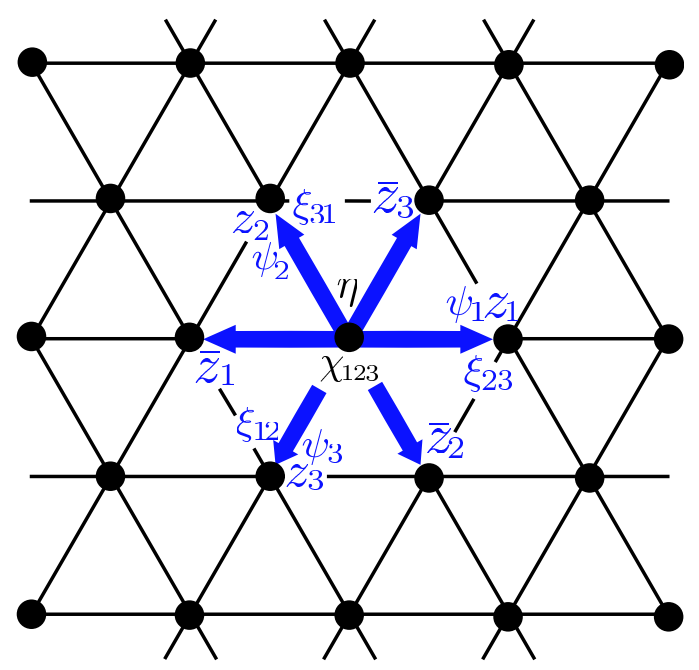

(a) $\mathbf{e}_{3}=-\mathbf{e}_{1}-\mathbf{e}_{2}$

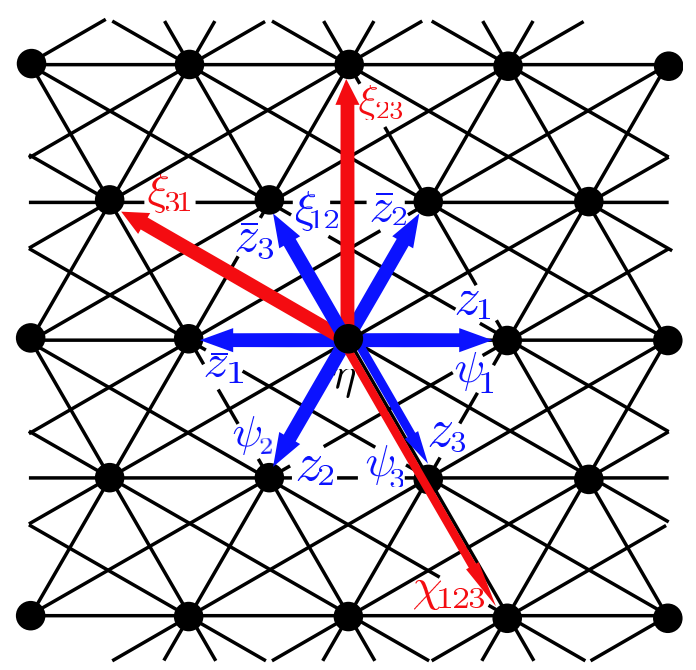

(b) $\mathbf{e}_{3}=\mathbf{e}_{1}+\mathbf{e}_{2}$

Figure 5: The lattice structure and the field configuration of the formulations [2-2] and [2-3]. The left figure (a) expresses the lattice structure and the field configurations for the case of $p=q=-1$ where there are two remaining supersymmetries. The right figure (b) expresses the case of $p=q=1$, where there is one remaining supersymmetry. For simplicity, we have chosen $a_{1}=a_{2}=a_{3}$ for both the cases.

This is the case of $p=q=-1$ and we again assume all $a_{m}$ are finite. Since $p$ and $q$ are negative, $\theta_{2}$ and $\theta_{3}$ are in the region (3.31), thus the lattice is triangular in general. Looking at Table $1, \chi_{123}$ turns out to be a singlet, so that this lattice theory possesses two conserved supercharges. Furthermore, in this formulation all fermions live on links of the triangles and there are no "diagonal" links. In fact, $\eta$ and $\chi_{123}$ live on sites, and $z_{m}$ and $\phi_{m}(m=1,2,3)$ are link variables in the directions of $\hat{\mu}_{m}$ given by (3.36). Also $\xi_{23}, \xi_{31}$ and $\xi_{12}$ live on the links, in the directions of $-\hat{\mu}_{1},-\hat{\mu}_{2}$ and $-\hat{\mu}_{3}$, respectively. (See (a) of Fig. 5.)

Using the same logic as in [2-1], we can show that the bosonic fields behave as a gauge field and six scalar fields in the naive continuum limit and the continuum theory is again expected to be two-dimensional $\mathcal{N}=(4,4)$ supersymmetric gauge theory.

$[2-3] \mathbf{e}_{3} \notin\left\{0,-\mathbf{e}_{1},-\mathbf{e}_{2},-\mathbf{e}_{1}-\mathbf{e}_{2}\right\}$

For any other rational values of $p$ and $q$ except for the special cases $(p, q)=(0,0)$, $(-1,0),(0,-1)$ and $(-1,-1), \eta$ is the only scalar fermion. The orbifolded lattice theories will then have only one preserved supersymmetry. The lattice structure is less symmetric than the above case and there are several "diagonal" link variables in general. As a simple example, consider the case of $p=q=1\left(\mathbf{e}_{3}=\mathbf{e}_{1}+\mathbf{e}_{2}\right)$. (See (b) of Fig. 5.) In this case, Lorentz invariance in the continuum forces the set $\left\{\hat{\mu}_{m}\right\}$ to be in the region (3.30). Now, $\xi_{12}$ and $\xi_{31}$ live on the diagonal links $\left(\mathbf{k}, \mathbf{k}-\mathbf{e}_{1}-\mathbf{e}_{2}\right)$ and $\left(\mathbf{k}, \mathbf{k}-\mathbf{e}_{3}-\mathbf{e}_{1}\right)$, respectively, and $\chi_{123}$ lives on the links $\left(\mathbf{k}, \mathbf{k}+2 \mathbf{e}_{1}\right)$. For the other fields, $\eta$ sits on sites, while $z_{m}, \bar{z}_{m}, \psi_{m}$ 
and $\xi_{23}$ live on the ordinary links. Using the same argument as above, we can show that the real components of $z_{m}$ and a linear combination of the imaginary components of $z_{m}$ become real scalar fields, and the other linearly independent components of the imaginary parts of $z_{m}$ form a vector field in the continuum limit. Thus the naive continuum limit is again two-dimensional $\mathcal{N}=(4,4)$ supersymmetric gauge theory.

\subsubsection{Relation between $N_{\text {shift }}=2$ and $N_{\text {shift }}=3$}

In this short subsection, we will mention an interesting connection between the cases of $N_{\text {shift }}=2$ and $N_{\text {shift }}=3$ discussed in sections 3.2.1 and 3.2.2, respectively.

In the case of $N_{\text {shift }}=3$, the space-time lattice is uniquely determined by (3.36) and (3.37) for given values of $(p, q)$ and $\left\{a_{m}\right\}$, where we assumed that the values of $a_{m}$ are all finite. However, we can easily see that these formulae can be applied even for the case where one of the $a_{m}$ go to infinity. Indeed, if we take the limit of $a_{3} \rightarrow \infty$ with $p, q \neq 0$, we obtain $\tan \theta_{2}= \pm \infty$ and $\tan \theta_{3}=\mp \frac{q a_{2}}{p a_{1}}$, where the upper sign is for $p, q>0$ and the lower sign is for $p, q<0$. This means that the two vectors $\hat{\mu}_{1}$ and $\hat{\mu}_{2}$ are orthogonal to each other and the direction of $\hat{\mu}_{3}$ is the same as $\mathbf{e}_{3}$ given by the linear relation (3.18). Furthermore, one can explicitly show that the combinations $\gamma_{m} a_{m} \hat{\mu}_{m}$ satisfy the relation (3.34). Then, recalling the definition of the shift (3.24), we conclude that the case of $N_{\text {shift }}=3$ contains the case of $N_{\text {shift }}=2$ as a special case. This result is true also for the cases of $p=0$ or $q=0$. In fact, (3.36) and (3.37) can be applied even then and we can safely take the limit of $a_{3} \rightarrow \infty$ for the case of $p=0$ and $q \neq 0$, and $a_{1} \rightarrow \infty$ for the case of $p \neq 0$ and $q=0$. When $p=q=0$, the theory becomes automatically [1-1] for any value of $a_{3}$.

As could have been expected intuitively, we thus find that all 2-shift solutions are just special cases of the general 3-shift solutions.

\section{Conclusions}

Following refs. [2, 3], we have considered the dimensionally reduced theories of fourdimensional $\mathcal{N}=1 \mathrm{SYM}$ theory and six-dimensional $\mathcal{N}=1 \mathrm{SYM}$ theory, and viewed them as "mother theories" for orbifolded lattice field theories. We have given what we believe is a complete classification of all possible lattice gauge theories in dimensions larger than or equal to two that can be constructed from these mother theories by the orbifolding procedure given in [2, 3]. We have imposed on the lattice theories that they have at least one preserved scalar supercharge, and that they become Lorentz invariant in the naive continuum limit.

Starting with the mother theory with four supercharges we have found that there is only one lattice formulation possible by this route. Its continuum limit is two-dimensional $\mathcal{N}=(2,2)$ supersymmetric gauge theory. This formulation is identical to what was given in [2]. We have thus shown that this formulation is unique.

On the other hand, starting with a mother theory with eight supercharges, there are many more possibilities. One can construct both three-dimensional and two-dimensional lattice theories in this case. We have found that the three-dimensional theory is again unique, and it coincides with the one given in [3]. For the two-dimensional theories, 
however, one can construct infinitely many lattice formulations labelled by two rational numbers $p$ and $q$. We have shown that they can be classified into seven categories by the number of remaining supersymmetries and the structure of the lattice. Five of these have two preserved scalar supercharges; the others have one. In the naive continuum limit, these formulations yield the same theory: two-dimensional $\mathcal{N}=(4,4)$ supersymmetric gauge theory. The five formulations with two supersymmetries, [1-1], [1-2], [1-3], [2-1] and $[2-2]$ are in a sense cousins. In fact, we can reach these formulations by tuning one of the fermions $\psi_{m}, \xi_{m n}$ and $\chi_{123}$ to be a singlet under the $U(1)$ symmetries. In these formulations, the space-time lattices are highly symmetric and form simple tilings of the two-dimensional plane. On the other hand, the lattice structures of the theories with one scalar supercharge, [1-4] and [2-3], are less symmetric. Therefore, even if the continuum limit is the same at tree level, one might prefer those lattice formulations that are closer to continuum Lorentz invariance already at finite lattice spacings. In this paper we have insisted on Lorentz invariance in the continuum limit. Since the lattice theories in question have at least one preserved supercharge at all lattice spacings it could be interesting to consider what types of continuum theories might emerge if one relaxes this condition.

Acknowledgement: We thank S. Hirano, I. Kanamori, K. Ohta, H. Suzuki, and T. Takimi for useful discussions. S.M. also acknowledges support from JSPS Postdoctoral Fellowship for Research Abroad.

\section{A. Useful Formulae}

In this appendix, we briefly review the orbifold projection and summarize some useful formulae. Let us consider a matrix theory (mother theory) and an adjoint field $\Phi$ in it, transforming as $\Phi \rightarrow g^{-1} \Phi g$ under the $U\left(k N^{n}\right)$ "gauge" symmetry. We also assume that this theory is invariant under a "global" symmetry $R$. In our case, $R=S O(4) \times U(1)$ for (2.1) and $R=S O(6) \times U(1)$ for (3.1). Suppose that $R$ contains $U(1)$ subgroups, $U(1)^{n}$, and $\Phi$ carries integer charges $\left(q_{1}, \cdots, q_{n}\right)\left(q_{a} \in \mathbb{Z}\right)$.

Under these assumptions, we consider a $Z_{N}^{n}$ symmetry generated by

$$
\gamma_{a}: \Phi \rightarrow \omega^{-q_{a}} \Omega_{a}^{-1} \Phi \Omega_{a}, \quad(a=1, \cdots, n)
$$

where $\omega=e^{2 \pi i / N}$ and

$$
\Omega_{a} \equiv 1_{k} \otimes \underbrace{1_{N} \otimes 1_{N}}_{a-1} \otimes U \otimes \underbrace{1_{N} \otimes 1_{N}}_{n-a}, \quad U \equiv \operatorname{diag}\left(\omega^{1}, \cdots, \omega^{N}\right) .
$$

Using $\gamma_{a}$, we can define a projection operator,

$$
P \equiv \frac{1}{N^{n}} \sum_{k_{1}, \cdots, k_{n}=1}^{N} \gamma_{1}^{k_{1}} \cdots \gamma_{n}^{k_{n}}
$$


Using the relation

$$
U^{-1} V U=\omega V, \quad V \equiv\left(\begin{array}{cccc}
0 & 1 & & \\
& \ddots & \ddots & \\
& & 0 & 1 \\
1 & & & 0
\end{array}\right)
$$

we can easily show that the projected matrix by $P$ can be expressed as

$$
\Phi=\sum_{\mathbf{m} \in \mathbb{Z}_{N}^{n}} \tilde{\Phi}(\mathbf{m}) \otimes U^{m_{1}} V^{q_{1}} \otimes \cdots \otimes U^{m_{n}} V^{q_{n}}
$$

or equivalently,

$$
\Phi=\sum_{\mathbf{k} \in \mathbb{Z}_{N}^{n}} \Phi(\mathbf{k}) \otimes E_{\mathbf{k}, \mathbf{k}+\mathbf{q}}
$$

where $\tilde{\Phi}(\mathbf{m})$ and $\Phi(\mathbf{k})$ are $k \times k$ matrices and we have defined

$$
E_{\mathbf{k}, \mathbf{l}}=E_{k_{1}, l_{1}} \otimes \cdots \otimes E_{k_{n}, l_{n}}
$$

with

$$
\left(E_{l, m}\right)_{i j} \equiv \delta_{l i} \delta_{m j} .
$$

The orbifold projection is defined by restricting fields in the mother theory to those which are invariant by the operation of $P$. Then, by construction, the orbifolded action is obtained by substituting (A.4) into the action of the mother theory. The orbifolded actions (2.16) and (3.12) are obtained by this procedure. In calculating the orbifolded action, the relation,

$$
E_{\mathbf{k}, \mathbf{l}} E_{\mathbf{m}, \mathbf{n}}=\delta_{\mathbf{l} \mathbf{m}} E_{\mathbf{k}, \mathbf{n}}
$$

is quite useful, which directly comes from the relation,

$$
E_{i, j} E_{k, l}=\delta_{j k} E_{i, l}
$$

\section{B. Supersymmetry Transformations}

In this appendix, we derive the explicit supersymmetry transformations of lattice theories constructed in this paper. We concentrate on the theories that are derived from the mother theory with eight supercharges, since it is this case which leads to new lattice formulations. The derivation for the other case (with four supercharges) is completely parallel.

Our treatment builds heavily on the very clear discussion in ref. [3]. We thus start with the supersymmetry transformations of the mother theory, and rewrite the action (3.1) as

$$
S_{m}=\frac{1}{g^{2}} \operatorname{Tr}_{N}\left(\frac{1}{4} v_{\alpha \beta}^{2}-\frac{i}{2} \operatorname{tr}_{2}\left(\tau_{2} \Psi^{T} C \bar{\Sigma}_{\alpha}\left[v_{\alpha}, \Psi\right]\right)\right)
$$


where $v_{\alpha \beta} \equiv i\left[v_{\alpha}, v_{\beta}\right], \operatorname{tr}_{2}$ denotes the trace over $2 \times 2$ matrix, and $\Psi$ is defined by

$$
\Psi \equiv\left(\psi, C \bar{\psi}^{T}\right)
$$

with a "charge conjugation matrix" $C$ satisfying

$$
C^{\dagger} \bar{\Sigma}_{m} C=\bar{\Sigma}_{m}^{T}, \quad C=C^{\dagger}=C^{-1}=-C^{T} .
$$

Note that, in this notation, the configuration (3.5) corresponds to

$$
\Psi=\left(\begin{array}{cc}
\eta & -i \chi_{123} \\
\xi_{23} & -i \psi_{1} \\
\xi_{31} & -i \psi_{2} \\
\xi_{12} & -i \psi_{3}
\end{array}\right)
$$

where we have used the representation,

$$
C=\left(\begin{array}{cccc}
0 & -i & 0 & 0 \\
i & 0 & 0 & 0 \\
0 & 0 & 0 & i \\
0 & 0 & -i & 0
\end{array}\right)
$$

One can easily check that (B.1) coincides with (3.1). In this notation, the supersymmetric transformation can be expressed compactly as

$$
\begin{aligned}
\delta v_{\alpha} & =\operatorname{tr}_{2}\left(\tau_{2} \kappa^{T} C \bar{\Sigma}_{\alpha} \Psi\right) \\
\delta \Psi & =-i v_{\alpha \beta} \Sigma_{\alpha \beta} \kappa,
\end{aligned}
$$

where $\kappa$ is a constant Grassmann parameter with the form of a $4 \times 2$ matrix.

We recall that the remaining supercharges on the lattice should correspond to fermions that have zero $U(1)$ charges. Furthermore, the supersymmetry parameter matrix $\kappa$ has the same structure as $\Psi$. We see that the supersymmetry transformations of the orbifolded theory therefore can be obtained by restricting $\kappa$ correspondingly, followed by the orbifolding projection. In the following, we derive those transformations that leave invariant the actions of the two-dimensional theories [1-2] and [2-1] $\left(\mathbf{e}_{1}+\mathbf{e}_{3}=0\right)$, and [1-3] and [2-1] $\left(\mathbf{e}_{1}+\mathbf{e}_{2}+\mathbf{e}_{3}=0\right)$ discussed in the section 3 . For the supersymmetry transformations of the three-dimensional theory and the two-dimensional theory [1-1], see ref. [3]. Those of the theories [1-4] and [2-3] are essentially the same as the three-dimensional theory, and we do not display them explicitly here.

\section{B.1 Supersymmetry transformations of the models 1-2 and 2-1}

In these cases, the $U(1)$ charges of $\eta$ and $\xi_{31}$ have $U(1)$ are zero. We thus fix the supersymmetry parameter matrix as

$$
\kappa=\left(\begin{array}{cc}
\kappa_{1} & 0 \\
0 & 0 \\
\kappa_{2} & 0 \\
0 & 0
\end{array}\right) .
$$


Substituting this into (B.6), we obtain

$$
\begin{aligned}
\delta z_{1} & =-2 i \kappa_{1} \psi_{1}, & \delta \bar{z}_{1} & =2 i \kappa_{2} \psi_{3}, \\
\delta z_{2} & =-2 i \kappa_{1} \psi_{2}+2 i \kappa_{2} \chi_{123}, & \delta \bar{z}_{2} & =0 \\
\delta z_{3} & =-2 i \kappa_{1} \psi_{3}, & \delta \bar{z}_{3} & =-2 i \kappa_{2} \psi_{1}, \\
\delta \eta & =\frac{i}{4} \sum_{m=1}^{3}\left[z_{m}, \bar{z}_{m}\right] \kappa_{1}+\frac{i}{2}\left[z_{1}, z_{3}\right] \kappa_{2}, & \delta \xi_{31} & =\frac{i}{2}\left[\bar{z}_{3}, \bar{z}_{1}\right] \kappa_{1}-\frac{i}{4}\left(\left[z_{1}, \bar{z}_{1}\right]-\left[z_{2}, \bar{z}_{2}\right]+\left[z_{3}, \bar{z}_{3}\right]\right) \kappa_{2} \\
\delta \xi_{23} & =\frac{i}{2}\left[\bar{z}_{2}, \bar{z}_{3}\right] \kappa_{1}+\frac{i}{2}\left[z_{1}, \bar{z}_{2}\right] \kappa_{2}, & \delta \xi_{12} & =\frac{i}{2}\left[\bar{z}_{1}, \bar{z}_{2}\right] \kappa_{1}+\frac{i}{2}\left[z_{3}, \bar{z}_{2}\right] \kappa_{2}, \\
\delta \psi_{m} & =0, & \delta \chi_{123} & =0 .
\end{aligned}
$$

Correspondingly, we can define two supercharges $Q_{1}$ and $Q_{2}$ as

$$
\delta=2 i \kappa_{1} Q_{1}+2 i \kappa_{2} Q_{2}
$$

which satisfy $Q_{1}^{2}=Q_{2}^{2}=0$ on-shell. In order to make the nilpotency satisfy off-shell, we introduce an auxiliary field $d$ and modify the transformations of $\eta$ and $\xi_{31}$ as

$$
\begin{aligned}
\delta \eta & =\left(\frac{i}{4} \sum_{m=1}^{3}\left[z_{m}, \bar{z}_{m}\right]-2 i d\right) \kappa_{1}+\frac{i}{2}\left[z_{1}, z_{3}\right] \kappa_{2}, \\
\delta \xi_{31} & =\frac{i}{2}\left[\bar{z}_{3}, \bar{z}_{1}\right] \kappa_{1}+\left(-\frac{i}{4}\left(\left[z_{1}, \bar{z}_{1}\right]-\left[z_{2}, \bar{z}_{2}\right]+\left[z_{3}, \bar{z}_{3}\right]\right)+2 i d\right) \kappa_{2},
\end{aligned}
$$

where the transformation of $d$ is

$$
\delta d=-\frac{i}{4} \sum_{m=1}^{3}\left[\psi_{m}, \bar{z}_{m}\right] \kappa_{1}+\frac{i}{4}\left(\left[z_{1}, \psi_{3}\right]-\left[z_{3}, \psi_{1}\right]-\left[\chi_{123}, \bar{z}_{2}\right]\right) \kappa_{2}
$$

Then $Q_{1}^{2}=Q_{2}^{2}=\left\{Q_{1}, Q_{2}\right\}=0$. The transformations (B.8) are those of the mother theory. The corresponding supersymmetry transformations of the lattice theory are obtained by substituting the expansion (3.11) into (B.8). It is tedious but straightforward to check that they indeed leave the lattice theory invariant.

\section{B.2 Supersymmetry transformations of the models 1-3 and 2-2}

In these cases, the $U(1)$ charges of $\eta$ and $\chi_{123}$ are zero, and we therefore fix $\kappa$ to be

$$
\kappa=\left(\begin{array}{cc}
\kappa_{1} & -i \kappa_{2} \\
0 & 0 \\
0 & 0 \\
0 & 0
\end{array}\right)
$$


Substituting this into (B.6), we obtain

$$
\begin{aligned}
\delta z_{l} & =-2 i \kappa_{1} \psi_{l}+i \kappa_{2} \sum_{m, n=1}^{3} \epsilon_{l m n} \xi_{m n}, & \delta \bar{z}_{m} & =0 \\
\delta \eta & =\frac{i}{4} \sum_{m=1}^{3}\left[z_{m}, \bar{z}_{m}\right] \kappa_{1}, & \delta \chi_{123} & =\frac{i}{4} \sum_{m=1}^{3}\left[z_{m}, \bar{z}_{m}\right] \kappa_{2}, \\
\delta \psi_{l} & =\frac{i}{4} \sum_{m, n=1}^{3} \epsilon_{l m n}\left[\bar{z}_{m}, \bar{z}_{n}\right] \kappa_{2}, & \delta \xi_{m n} & =\frac{i}{2}\left[\bar{z}_{m}, \bar{z}_{n}\right] \kappa_{1} .
\end{aligned}
$$

Again, we can define two supercharges $Q_{1}$ and $Q_{2}$ by $(\overline{\mathrm{B} .9})$, and we can again make them nilpotent off-chell by introducing an auxiliary field $d$ in the transformations of $\eta$ and $\chi_{123}$ :

$$
\begin{aligned}
\delta \eta & =\left(\frac{i}{4} \sum_{m=1}^{3}\left[z_{m}, \bar{z}_{m}\right]-2 i d\right) \kappa_{1}, \\
\delta \chi_{123} & =\left(\frac{i}{4} \sum_{m=1}^{3}\left[z_{m}, \bar{z}_{m}\right]-2 i d\right) \kappa_{2},
\end{aligned}
$$

where the transformation of $d$ is defined as

$$
\delta d=-\frac{i}{4} \sum_{m=1}^{3}\left[\psi_{m}, \bar{z}_{m}\right] \kappa_{1}+\frac{i}{8} \sum_{l, m, n=1}^{3} \epsilon_{l m n}\left[\xi_{m n}, \bar{z}_{l}\right] \kappa_{2} .
$$

One can explicitly check that $Q_{1}$ and $Q_{2}$ now satisfy $Q_{1}^{2}=Q_{2}^{2}=\left\{Q_{1}, Q_{2}\right\}=0$. Again, the supersymmetry transformations for the lattice theory are obtained by substituting the expansion (3.11) into (B.13).

\section{References}

[1] D. B. Kaplan, E. Katz and M. Unsal, Supersymmetry on a spatial lattice, JHEP 05 (2003) 037 hep-lat/0206019.

[2] A. G. Cohen, D. B. Kaplan, E. Katz and M. Unsal, Supersymmetry on a Euclidean spacetime lattice. I: A target theory with four supercharges, JHEP 08 (2003) 024 hep-lat/0302017.

[3] A. G. Cohen, D. B. Kaplan, E. Katz and M. Unsal, Supersymmetry on a Euclidean spacetime lattice. II: Target theories with eight supercharges, JHEP 12 (2003) 031 hep-lat/0307012.

[4] D. B. Kaplan and M. Unsal, A Euclidean lattice construction of supersymmetric Yang-Mills theories with sixteen supercharges, JHEP 09 (2005) 042 hep-lat/0503039.

[5] M. G. Endres and D. B. Kaplan, Lattice formulation of (2,2) supersymmetric gauge theories with matter fields, JHEP 10 (2006) 076 hep-lat/0604012.

[6] S. Catterall and S. Karamov, Exact lattice supersymmetry: the two-dimensional $N=2$ Wess-Zumino model, Phys. Rev. D65 (2002) 094501 hep-lat/0108024.

[7] S. Catterall and S. Karamov, A two-dimensional lattice model with exact supersymmetry, Nucl. Phys. Proc. Suppl. 106 (2002) 935-937 hep-lat/0110071. 
[8] S. Catterall and S. Ghadab, Lattice sigma models with exact supersymmetry, JHEP 05 (2004) 044 hep-lat/0311042].

[9] S. Catterall, Lattice supersymmetry and topological field theory, JHEP 05 (2003) 038 hep-lat/0301028.

[10] S. Catterall, Lattice formulation of $N=4$ super Yang-Mills theory, JHEP 06 (2005) 027 hep-lat/0503036.

[11] S. Catterall, Simulations of $N=2$ super Yang-Mills theory in two dimensions, JHEP 03 (2006) 032 hep-lat/0602004.

[12] A. D'Adda, I. Kanamori, N. Kawamoto and K. Nagata, Twisted superspace on a lattice, Nucl. Phys. B707 (2005) 100-144 hep-lat/0406029].

[13] A. D'Adda, I. Kanamori, N. Kawamoto and K. Nagata, Exact extended supersymmetry on a lattice: Twisted $N=2$ super Yang-Mills in two dimensions, Phys. Lett. B633 (2006) 645-652 hep-lat/0507029.

[14] F. Bruckmann and M. de Kok, Noncommutativity approach to supersymmetry on the lattice: SUSY quantum mechanics and an inconsistency, Phys. Rev. D73 (2006) 074511 hep-lat/0603003.

[15] F. Bruckmann, S. Catterall and M. de Kok, A critique of the link approach to exact lattice supersymmetry, Phys. Rev. D75 (2007) 045016 hep-lat/0611001.

[16] F. Sugino, A lattice formulation of super Yang-Mills theories with exact supersymmetry, JHEP 01 (2004) 015 [hep-lat/0311021].

[17] F. Sugino, Super Yang-Mills theories on the two-dimensional lattice with exact supersymmetry, JHEP 03 (2004) 067 [hep-lat/0401017.

[18] F. Sugino, Various super Yang-Mills theories with exact supersymmetry on the lattice, JHEP 01 (2005) 016 hep-lat/0410035.

[19] F. Sugino, Two-dimensional compact $N=(2,2)$ lattice super Yang-Mills theory with exact supersymmetry, Phys. Lett. B635 (2006) 218-224 hep-lat/0601024.

[20] M. Unsal, Compact gauge fields for supersymmetric lattices, JHEP 11 (2005) 013 hep-lat/0504016.

[21] M. Unsal, Twisted supersymmetric gauge theories and orbifold lattices, JHEP 10 (2006) 089 hep-th/0603046.

[22] N. Arkani-Hamed, A. G. Cohen and H. Georgi, (De)constructing dimensions, Phys. Rev. Lett. 86 (2001) 4757-4761 hep-th/0104005.

[23] J. Giedt, E. Poppitz and M. Rozali, Deconstruction, lattice supersymmetry, anomalies and branes, JHEP 03 (2003) 035 hep-th/0301048].

[24] J. Giedt, Non-positive fermion determinants in lattice supersymmetry, Nucl. Phys. B668 (2003) 138-150 hep-lat/0304006.

[25] T. Onogi and T. Takimi, Perturbative study of the supersymmetric lattice theory from matrix model, Phys. Rev. D72 (2005) 074504 [hep-lat/0506014.

[26] K. Ohta and T. Takimi, Lattice formulation of two dimensional topological field theory, hep-lat/0611011. 
[27] H. Fukaya, I. Kanamori, H. Suzuki, M. Hayakawa and T. Takimi, Note on massless bosonic states in two-dimensional field theories, Prog. Theor. Phys. 116 (2007) 1117-1129 hep-th/0609049.

[28] J. Giedt, Deconstruction and other approaches to supersymmetric lattice field theories, Int. J. Mod. Phys. A21 (2006) 3039-3094 hep-lat/0602007.

[29] J. M. Rabin, HOMOLOGY THEORY OF LATTICE FERMION DOUBLING, Nucl. Phys. B201 (1982) 315.

[30] P. Becher and H. Joos, The Dirac-Kahler Equation and Fermions on the Lattice, Zeit. Phys. C15 (1982) 343.

[31] H. Suzuki and Y. Taniguchi, Two-dimensional $N=(2,2)$ super Yang-Mills theory on the lattice via dimensional reduction, JHEP 10 (2005) 082 hep-lat/0507019.

[32] P. H. Damgaard and S. Matsuura, in preparation.

[33] T. Eguchi and H. Kawai, Reduction of Dynamical Degrees of Freedom in the Large $N$ Gauge Theory, Phys. Rev. Lett. 48 (1982) 1063.

[34] P. Orland, VOLUME REDUCTION OF LATTICE GAUGE SYSTEMS AT FINITE N, Phys. Lett. B134 (1984) 95. 